




\section{ARTICLE IX.}

Researches concerning the Periodical Meteors of August and November. By Sears C. Walker, A. P. S. Read January 15, 1841.

\section{$\S$ I. Of the Relative Velocities of shooting Stars.}

The discovery of the existence of a radiant, or its antipode, the convergent point for the relative paths of the shooting stars composing the splendid spectacle of November 12th, 1833, and its confirmation on several subsequent, but less brilliant displays of these bodies, has opened the field for fresh researches concerning their geometrical relations. The earliest attempt to deduce the necessary inferences from such a discovery was made by Prof. Olmsted, ${ }^{1}$ shortly after the great shower of 1833 . In this inquiry he was followed, in 1834, by Prof. Twining ${ }^{2}$ and Mr. Espy ${ }^{3}$; in 1835, by Arago ${ }^{4}$, Biot ${ }^{5}$ and others; and, in 1836 and 1837, more concisely, by Quetelet ${ }^{6}$ and Olbers ${ }^{7}$, and, subsequently, by Mr. Herrick ${ }^{8}$ and Prof. Lovering. ${ }^{9}$ Finally, in 1839, a full and systematic inquiry on the subject was instituted by Prof. Erman, ${ }^{10} \mathrm{Jr}$., of Berlin. An abstract of the

1 Silliman's Journal, vol. xxvi., p. 144. See also subsequent volumes.

${ }^{2}$ Idem. vol. xxvi., art. viii.

${ }^{3}$ Journal Franklin Institute, vol. xv., p. 9.

${ }^{4}$ Annuaire du Bureau des Longitudes, $1836 . \quad{ }^{5}$ Sill. vol. xxxii., p. 181.

${ }^{6}$ Quetelet's Catalogue des Principales apparitions d'étoiles filantes. Nouveaux Mémoires de l'Académie, \&c., de Bruxelles, 1839. Also Annuaire de l'Observatoire de Bruxelles, 1837.

7 Die Sternschnuppen, Schumacher's Jahrbuch for 1838, p. 319, note. Also Schumacher's Astronomische Nachrichten, Nos. 372, 384 .

${ }^{8}$ Sill. vol. xxxiii., art. xx., and vol. xxxv., art. xix.

${ }^{9}$ Idem. vol. xxxv., art, $\mathrm{x}$.

${ }^{10}$ Astr. Nachr., Nos. 385, 390, 402, and 404. 
limits assigned by this author for the elliptic elements of some of these bodies, considered as asteriods, has been published in the proceedings of this Society for August 21st, 1840. An examination of Professor Erman's analysis having led to the conclusion that his limits for these elements are too restricted, and that more simple formulæ might be obtained by adopting instead of the earth's actual velocity, the well known Gaussian constant as the unit of linear velocities, induced me to undertake the discussion afresh. In doing so, however, it is proper to remark, as must be obvious to every one, that the nature of the subject is such as to deprive the discussion of that demonstrative character which distinguishes the results of astronomy proper. Such a circumstance, however, should not deter us from aiming at the greatest precision in our knowledge of the geometrical relations of these small bodies, which the nature of the case permits.

The principal data which the theory of shooting stars derives from observation are their relative velocities and directions as seen by an eye in motion, and the dates of remarkable showers, or brilliant meteoric displays. These data, if furnished with precision, are sufficient for the completion of their theory, considered as cosmical bodies. In the case of a newly discovered planet or comet, a single observation furnishes only a geocentric position, the distance, relative velocity, and direction of motion, being as yet unknown. Hence three successive positions, at known intervals, are required in order to determine, by Kepler's and Newton's laws, the (geocentric or heliocentric) distance, velocity and direction of motion, at one of the three dates,-from which all the elements of the elliptic orbit of the planet or comet may be derived. When we consider the precision of observation, and the length of elapsed time, which are requisite for determining the path of a planet or comet, it will appear surprising at first, that enough should ever be known concerning the geometrical relations of a body, which appears for a moment and then vanishes for ever, to enable us even to form a conjecture concerning its true motion in the heavens for an indefinite period past and to come. There are, however, several important advantages in the case of shooting stars which do not present themselves in a single observation of a newly discovered planet or comet. The shooting star or asteroid is necessarily within a few seconds of its node, and its heliocentric radius vector differs from that of the spectator by a quantity so small as to be safely neglected in computations for the approximate elements of its orbit. A similar remark applies to the heliocentric longitude of the observer 
and asteroid, which may be regarded as common. By the ordinary computations for the transfer of co-ordinates from the centre of the earth to the position of the spectator, or quite as well by neglecting quantities so small, it is always possible to determine three data for the orbit of every meteor that is seen, namely, the node, radius vector, and time of passing the node. To complete the six elements it is only necessary to know, at the same time, three other quantities, the asteroid's true or relative velocity in space, and its direction with reference to two given planes, the equator or ecliptic, for instance, and a secondary to the same. The determination of the first of these three requisites, the relative velocity of shooting stars, was first undertaken in 1798, by two students of Gottingen, Brandes and Benzenberg, ${ }^{11}$ and was pursued with a zeal which terminated only in the death of the former. A notice of their labours has been given by Olbers, and by Quetelet. An abstract by Professor Loomis of the results obtained by Brandes, in 1823, may be found in Silliman's Journal, ${ }^{12}$ giving the results of corresponding observations in Breslaw and its vicinity. In 1824, Quetelet ${ }^{13}$ and others in Brussels and its neighbourhood engaged in similar researches. On the memorable occasion of the display of November 12 th, 1833 , among the myriads of meteors seen, one only was known to have been beheld at several places. It was distinguished by its extraordinary size and brilliancy, and by the duration (ten minutes at least) of its train, which, after assuming various serpentine shapes, "terminated in a luminous nebula of several times the diameter of the moon, floating onwards with a velocity greater than that of the clouds." Mr. Twining, ${ }^{14}$ after a full discussion of all the facts connected with this meteor, concludes that its apparent path was about forty-eight 'geographical miles, and its duration about three seconds, making a mean relative velocity of about sixteen miles per second. Corresponding observations were made at Breslaw and its vicinity by Boguslawski and others, Nov. 13th, 1836, and August 9th, 1837. I have not been able to obtain the single results, and therefore quote the remark of Olbers, ${ }^{15}$ that results obtained for the November meteors of 1836 "show that the periodical meteors also have the same height and relative velocity as the ordinary shooting stars hitherto

11 Versuche, die entfernung, \&c., der Sternschnuppen zu bestimmen. Hamburg, 1800.

Bestimmung der geographischen Länge durch Sternschnuppen, Von I. F. Benzenberg. Hamburg, 1802.

19 Vol. xxviii., p. $95 . \quad{ }^{13}$ Catalogue, \&c., p. 5.

14. Silliman, vol. xxvi., p. 46.

${ }^{15}$ Schumacher's Jahrbuch, for 1838, p. 322. Note. VIII. $-\mathrm{X}$ 
observed." Also, Quetelet ${ }^{16}$ remarks that Mr. Boguslawski obtained "results analogous" to those of his table for the relative velocities of the meteors of August 9th, 1837. On the 29th of August, 1838, the younger Littrow ${ }^{17}$ obtained corresponding observations of several meteors in Vienna and its vicinity. An effort by Mr. E. C. Herrick and others, in April, 1839, in New Haven, Middlebury, Williamstown, Cambridge, and other places, was unsuccessful for want of coincidences, like a similar attempt of Brandes and others in 1817. The method adopted in all instances is to prove the identity ${ }^{18}$ of the meteors seen at two different places. Then the space traversed, and the duration give the relative velocity. The Vienna observations of 1838 , for relative velocities, have not been fully reduced, the memorandums for duration not being complete. Such results, as far as obtained, are given in Table I., chiefly from Quetelet's Memoir on Shooting Stars. The results obtained by Twining, and the remarks quoted from Olbers and Quetelet are important in the present inquiry, as they show that the mean relative velocity, 18.3 geographical miles per second from all the results yet obtained, may be taken for a first approximation in estimating the elements of the elliptic orbits of those meteors or asteroids whose relative direction is known. It is much to be desired that Table I. should be farther extended, and, as an encouragement to enterprise in this department of meteorology, we have the high authority of Bessel,${ }^{19}$ who " doubts not that every desirable degree of perfection is attainable by observation, in so far as regards our knowledge of the geometrical relations of shooting stars."

On examining Table $I$. it will appear that the single results arrange themselves on both sides of the mean result 18.3 miles per second, with an average discrepancy of about 5.2 miles per second. As far as we can judge from so small a number of results, necessarily somewhat imperfect, it would seem that the mean relative velocity of shooting stars tends towards that of the earth in its orbit, namely, 16.4589 geographical miles (of 60 to a degree) per second, with

${ }^{16}$ Catalogue, \&c., p. 6. Note.

17 Annalen der K. K. Sternwarte in Wien, 1838. p. xviii.

18 See Loomis' Notice of Brandes' Memoir, above quoted, p. 98.

${ }^{19}$ Uber Sternschnuppen Astr. Nachr. 381, p. 50. "Ich zweifle nicht, dass die Kentniss der Sternschnuppen, in so fern von dell geometrischen Verhältnissen, die man daran wahrnehmers kann, die Rede ist, so vollständig gemacht werden kann, als man zu wünschen berechtigt ist." 
average discrepancies of less than one-third of that value. The first result is such as we should naturally expect, since, in the case of bodies moving with all varieties of directions and velocities, there must be a compensation of these velocities resolved each at the time of visibility in the direction of the observer's tangential motion. The second result-the smallness of the average discrepancy-if it leads to any conclusion at all, shows that the average true velocity of the meteors is small, or the mean discrepancy of the relative velocities would be greater. Lastly, this mean relative velocity of shooting stars is so great as to preclude the possibility of a terrestrial or lunar origin. Since it follows from the laws of gravity, according to the remark of Olbers, ${ }^{20}$ Laplace, ${ }^{21}$ Hassler, ${ }^{22}$ and others, that the mean relative velocity of a satellite of the earth, at its nearest possible approach to the observer, is only about 4.29 geographical miles, and its maximum in a re-entering orbit only 6.06 geographical miles. Hence all bodies of our system which, when visible to us, have a relative velocity beyond this limit, must be moving relatively to the earth in a hyperbolic orbit, and must, in a few hours, leave the earth's sphere of activity, and again become, as they must have been before, cosmical bodies. The grounds for the latter remark, that bodies having a relative velocity of 18.3 miles cannot have acquired the same by any force belonging to the atmosphere, nor by any volcanic or other explosive force in the earth or moon, are manifest. Indeed, the explosive force of gunpowder communicates a velocity of only a fourth of a geographical mile per second, ${ }^{23}$ and a velocity of seventy times that amount cannot be ascribed to any force known to exist in the earth or moon; and certainly no such force must, on account of this observed relative velocity alone, be presumed to exist, when other phenomena point to the sun's central force as amply sufficient to furnish such a relative velocity as the resultant of a cosmical body's true and the observer's known tangential motion.

${ }^{20}$ Jahrbuch, for 1837, p. 56 . Note.

${ }^{21}$ Systeme du Monde, L. iv. chap. v.

${ }^{22}$ Mem. Am. Phil. Soc. Vol. vi., P. II., Art. xv.

${ }^{23}$ Monat. Corr. June 1812, p. 564. The velocity of a twenty-four pound cannon-ball is stated by Lagrange at 1398 Paris feet per second. 
Table I.

Relative Second's Velocities of Meteors in Geographical Miles.

\begin{tabular}{|c|c|c|c|}
\hline Date. & $\begin{array}{c}\text { Designation. } \\
\text { of Meteor. }\end{array}$ & $\begin{array}{l}\text { Second's } \\
\text { velocity. } \\
x y .\end{array}$ & $\begin{array}{c}\text { Observer } \\
\text { and } \\
\text { Computer. }\end{array}$ \\
\hline$\underset{66}{1798 .}$ & $\begin{array}{l}a \\
b\end{array}$ & $\begin{array}{l}24 . \\
18 .\end{array}$ & Brandes. \\
\hline 1823. & a & 20. & 46 \\
\hline “6 & b & 24. & ، \\
\hline "6 & c & 32. & “ \\
\hline$\underset{66}{1824 .}$ & $\begin{array}{l}a \\
b\end{array}$ & $\begin{array}{l}15 . \\
22.8\end{array}$ & $\begin{array}{c}\text { Quetelet. } \\
66\end{array}$ \\
\hline ، & c & 13.5 & ، \\
\hline ، & d & 9. & ، \\
\hline ، & e & 15. & “، \\
\hline “ & $\mathrm{f}$ & 10.2 & “6 \\
\hline $\begin{array}{l}\text { 1833, November } 12 . \\
1836 \text {, November } 13 . \\
1837 \text {, August } 9 \text {. }\end{array}$ & $\begin{array}{c}\mathrm{a} \\
\mathrm{a} \mathrm{b}^{\mathrm{c}} \mathrm{d} \\
\text { unknown }\end{array}$ & $\begin{array}{l}16 . \\
\text { average } \\
\text { average }\end{array}$ & $\begin{array}{l}\text { Twining. } \\
\text { Boguslawski. }\end{array}$ \\
\hline Mean second's & \multicolumn{3}{|c|}{$18^{\mathrm{m}} \cdot 3 \pm 1^{\mathrm{m}} \cdot 4=1.112=\gamma$} \\
\hline
\end{tabular}

The mean value of Table $I$. is further confirmed, by considerations derived from the mean duration and mean length of the visible paths of meteors. Mr. Custodes $^{24}$ found, from ninety-eight shooting stars, seen 9th August, 1837, at Dusseldorf, that the mean duration of visibility was $1^{\prime \prime} 12 . " \prime 7$; that of twentyeight of the largest size $1^{\prime \prime} 45 . " \prime$ 9-none over 3." Benzenberg, from observations at various seasons of the year, concludes that the mean duration was more than 1." These observations were made by means of a clock marking thirds, (tertian-clock.) The length of the paths of the four meteors seen at Breslaw and at a station in its vicinity, are given by Olbers. ${ }^{25}$ Also those of ten coincidences at Vienna and Calvarienburg, August 29th, 1838, are given by the younger Littrow, ${ }^{26}$ both, as follows, in geographical miles of sixty to a degree.

$\begin{array}{cccc}\text { 1836, November 12, meteor a, visible path } & 5.96 \\ \text { “" } & \text { “ } & \text { b, } & 24.88 \\ \text { “ } & \text { “ } & \text { c, } & 32.88 \\ \text { “ } & \text { "6 } & \text { d, } & 43.52 \\ \text { 1838, August 29, } & \text { “ } & \text { a, } & 12.28 \\ \text { "6 } & \text { " } & \text { b, } & 29.28 \\ \text { “ } & \text { " } & \text { c, } & 17.20\end{array}$

${ }^{24}$ Schumacher's Jahrbuch, 1838, p. 324. $\quad{ }_{-2}^{25}$ Idem. p. 322, note. $\quad{ }^{26}$ Annalen, \&c. for 1838, p. xxi. 


\begin{tabular}{|c|c|c|c|}
\hline 1838, Aus & leteo & $\mathrm{d}$, visible path & 13.3 \\
\hline 6 & $" 6$ & $\mathrm{e}$ & 12.28 \\
\hline "6 & 66 & $\mathrm{f}$, & 31.26 \\
\hline 66 & 6 & $\mathrm{~g}$, & 20.84 \\
\hline “6 & “ & $\mathrm{h}$, & 26.44 \\
\hline “ & 6 & $\mathrm{i}$, & 0.36 \\
\hline$\because$ & "s & $\mathrm{k}$, & 13.16 \\
\hline & & 一 & - \\
\hline & & 14 mean & 22.64 \\
\hline & & - & - \\
\hline For mear & $1^{\prime \prime} .2$ & 1, mean velocity & 18.71 \\
\hline
\end{tabular}

Prof. Locke, of the Ohio Medical College, and Mr. Dwelle, made corresponding observations in Cincinnati, and its vicinity, in August, 1834. The results were published shortly afterwards, in the Cincinnati Gazette of that year. They found the average height about forty-five miles, the average velocity about twenty miles per second, and the average duration about $1^{s}$. They also noticed an unusual number of meteors about the 10th of August.

\section{\$II.-Of The Relative Directions of shooting Stars in Space.}

Division I.-Of the Shooting Stars segn on ordinary Nights.

The remaining requisite for the complete determination of the orbits of these asteroids is their relative tangential direction as seen by an observer in motion, both orbital and rotary; in other words, the apparent path and direction of the meteor as affected by annual and diurnal aberration. The observation of a single meteor's path gives no clue to the solution of the question. If, however, we mark on a globe the points of appearance and vanishing of a meteor, and connect them by a great circle, then the inclination of the plane of this circle to a line drawn from the centre of the globe to any given point may be measured; or, the problem may be solved analytically, and the inclination computed. Thus, on any occasion in which a great number of paths have been delineated, we may assume any point in the sphere as the trial point, and VIII. $-\mathrm{Y}$ 
compute the R. A. and Dec. of the points of nearest approach of this trial point to the several planes in which the observed paths are contained. Then, if there is any general tendency of the relative directions to one point in the sphere more than to another, this circumstance will be indicated by the position of the points of nearest approach, and the mean of these several positions will afford an approximate convergent point, from which the actual convergent point for the evening may be ascertained. As a trial point it appears most natural to commence with the antipode of the observer's tangential direction, this being the point towards which a tendency of the relative paths should naturally be impressed. Indeed, if we suppose the meteors moving with all varieties of true direction and velocity, a compensation of the latter should be expected to take place, as has been found to be the case with the former; and the outstanding, uncompensated position of the convergent point should be, as just stated, the point opposite the observer's true direction. The only observers, as far as I know, who have detected such a relative convergent point, on ordinary nights, are Mr. Fitch, of New Haven, ${ }^{27}$ who found this point to advance in the ecliptic from August to December, remaining always about $90^{\circ}$ in advance of the sun, or $90^{\circ}$ in arrear of the earth, and Mr. E. C. Herrick ${ }^{28}$ and others, who noticed the same phenomenon in April, 1839. Professor Forshey ${ }^{29}$ of Mississippi, who has given much attention to this subject, and C. L. Von Littrow, ${ }^{30}$ adjunct at the Imperial Observatory of Vienna, who nightly traced the paths of meteors for nearly a year, from 1837 to 1838, speak of the existence of a convergent point as being peculiar to the dates of August 9th-12th, and Nov. 11th-13th. Also, Olbers ${ }^{31}$ makes the prevalence of parallelism of paths and a radiant point in Leo the distinguishing feature of the November phenomenon of 1838 , these being present on the 13th, but not on the 12th of that year. Nor do I read of any other authors but Messrs. Fitch and Herrick, and their associates, who have discovered this motion. The a priori probability of the tendency towards such a convergent point, by the meteors seen on ordinary nights, is so great that it is next to a miracle that it should not occur. In case of its occurrence, the average discrepancy of the single results from

- $\quad{ }^{27}$ Sill. vol. xxxiii., p. 386.

${ }^{29}$ Mentioned in conversation, August, 1840.

${ }^{28}$ Letter received from Mr. Herrick.

astr. Nachr., 372.

3o Annalen, \&c., 1838, p. xvii. 
the theoretic convergent point, if well observed, would, like those of the single relative velocities from the observer's true velocity, afford us some clue towards the discovery of the mean true velocities of shooting stars seen on ordinary nights. The fact that Messrs. Fitch and Herrick detected the existence of this actual relative convergent point, conformable to the theoretic, leads to a conclusion similar to that derived from the table of relative velocities; namely, that the true velocity of the meteors is small compared with that of the ob. server, whence the greater prevalence of the theoretic convergent point. It is probable, then, that this tendency of a part of the meteors, on ordinary nights, towards the vicinity of the point opposite to the observer's direction of motion, continues throughout the year, and that on each night another portion of the meteors appears to be non-conformable, or sporadic, as they are termed by Olbers and Quetelet. Hence we infer that convergent meteors, so called on ordinary nights, must be those which move with small, and sporadic meteors those which move with greater true velocity. This circumstance has been noticed with respect to their relative angular velocities by Mr. Dutton. ${ }^{3.2}$ A consideration of the circumstances connected with the elements of elliptic motion of these asteroids leads to a classification of the orbits of the convergent and sporadic meteors seen on common nights. The former could not have the prevalent character of convergency towards the point opposite to the observer's direction of motion, without having their true velocity small. Then they must have their perihelia near the sun, perhaps inferior to Mercury, and must be near their aphelia, and have, therefore, pretty eccentric orbits, in order to reach the earth in their aphelia. The sporadic meteors may be justly considered as a class of asteroids superior to the convergent meteors, whether having their perihelia near the sun, and being very eccentric, with aphelia far superior to that of the earth, or having their perihelia not much inferior to that of the earth. In each of the respective cases they must have great true velocities, and, moving in all varieties of true direction, the average deflection of their relative directions from the point opposite the observer's direction is so great that they are called non-conformable or sporadic. It will be shown, in the sequel, that the conclusion drawn from the table of observed relative velocities, as well as from the existence of a convergent point, on common nights, opposite to the observer's true direction, namely, that a large portion of meteors seen on ordi- 
nary occasions are asteroids, having their perihelia more aggregated together as distance from the sun diminishes, is a priori rendered probable from the known analogies of the solar system. This general classification of these shooting stars, founded only on probable grounds, seems to be all the inference that we are able to draw from the existence of an actual convergent point on common nights, conformable to the theoretic, and from the facts known respecting their relative velocities. Of the particular elements of motion of the individuals seen, there are no grounds for forming even a conjecture. The simple circumstance of this compensation of their true directions, and true normal velocities, while it warrants the general conclusion that they are inferior asteroids, also leads to a belief that the planes of their orbits are promiscuous in position.

The impossibility of determining the relative direction of single meteors, on ordinary nights, by observations at a single station, and the sources of error pointed out chiefly by Bessel, ${ }^{33}$ in regard to duration, absolute time, position of point of appearance and vanishing, and the uncertainty whether the visible path to observers in different stations is common, must, even when several stations are employed, hinder, if not entirely frustrate, all our attempts to deduce the cosmical elements of motion of any one of the meteors seen on ordinary nights. Hence it would seem that if the elliptic elements of any one of these asteroids are ever to be known, it must be chiefly by means of corresponding observations on those extraordinary occasions when the occurrence of a new feature, not present on common nights, enables us to determine with precision their relative directions by observations at a single station.

\$ II.-Division 2.-Of the convergent Point of the relative Paths of Meteors on the Occasions of extraordinary Displays.

The ordinary number of meteors visible by a single observer is, according to Olbers, ${ }^{34}$ Quetelet ${ }^{35}$ Herrick ${ }^{36}$ and others, about eight per hour. Those occasions in which a greater number is seen are more or less extraordinary. In most instances of these extraordinary displays, another circumstance is noticed on careful observation. The relative directions or deflections from the anti-

${ }^{33}$ Uber Sternschnuppen,-already quoted.

${ }^{35}$ Catalogue, \&c., p. 12, 13.
${ }^{34}$ Jahrbuch, for 1838 , p. 325.

${ }^{36}$ Silliman, vol. xxxv., p. 172. 
pode of the observer's direction are not compensated as on ordinary nights, but there remains an outstanding uncompensated tendency towards a point more or less deflected from this antipode, indicating the prevalence of a true mean velocity, of greater or less magnitude, in a perpendicular to the observer's direction. The meteors which do not appear to tend towards this extraordinary convergent point are termed sporadic, and in such cases the term includes both classes of sporadic and convergent meteors of ordinary nights. It seems to me, in conformity with the opinion of Olbers, that the appearance of an extraordinary convergent point is a better and surer criterion of an extraordinary display of meteors than a moderate excess of numbers above the mean. Let us suppose that an observer on the earth's surface, in his annual and rotary motion, falls in with a portion of planetary space interspersed with these small bodies, separated by intervals of not many hundred miles, yet so far separated as to exhibit no tendency towards fixedness of relative position, like the particles of the single bodies themselves, or like the solids, liquids, or even gases, connected with the earth,- their mutual perturbations, owing to the smallness of their masses, not being much greater than those of the principal planets of the system. Let us suppose, however, that besides proximity in position in the system, the separate bodies have also common elements of motion, the discrepancies in the latter being of the same order as those of position relatively to the whole system, whether from common circumstances connected with their origin, or primitive projectile force. Then we should have all the phenomena of an extraordinary display of meteors, namely, unusual numbers, and unusual position of the convergent point of their relative paths. This position could readily be determined by the method already stated; and the mean relative direction in space of this flock of bodies could be ascertained by a single observer, at one station, with a precision which, but for their common relations, no corresponding observations at any variety of stations could afford. Then the only remaining element for the complete determination of the mean elliptic elements of this small system, or cluster of asteroids, would be the relative velocity of one of them, or their mean relative velocity, (the single velocities being nearly common by hypothesis.) In the absence of a complete determination of this velocity, if it were presumable that this mean value was analogous to that of Table I., then, since the mean error of the position of the convergent point is very small, and may be neglected, a system of elements derived from the adoption of the mean value in Table $\dot{I}$. would have vIII. -Z 
that degree of plausibility which naturally belongs to the statements quoted from Olbers and Quetelet, respecting the relative velocities of meteors in extraordinary showers, and the coincidence therewith of the single instance of relative velocity for one of the November meteors computed by Professor Twining. I shall here subjoin the principal data which we possess concerning the convergent point for the extraordinary displays of August 9th-11th and November 11th-13th. The angle of deflection of the former from the theoretic convergent point for isolated meteors is $39^{\circ} .4$; that of the latter only $8^{\circ} .2$, a quantity not much exceeding the probable error. So that one important criterion of extraordinary character is wanting in the November period, namely, a well marked and manifest deflection of their convergent point from that of isolated and promiscuous meteors. The convergent point of the "meteoric abundance," so called, of December 7th, 1838, was found by Mr. Herrick ${ }^{37}$ to be nearly the same as that of the August period. This would give a deflection of $104^{\circ}$ from the point opposite the observer's direction. As this phenomenon is not known to have presented itself before nor since, it may, perhaps, be considered as an example of an isolated cluster of these bodies having common elements of motion in a plane highly inclined to the ecliptic, in a period which we have no means of estimating, since the want of corresponding observations concerning the relative velocity of any one of these bodies leaves us in doubt how far these velocities were conformable with those of Table I. One consequence follows, however, from this high angle of deflection, that the mean normal velocity of the cluster could not have been less than that of the observer, and that the single bodies must have been superior planets. An attempt will be made to deduce the most plausible estimate of the elements of the asteroids of August and November, after discussing the circumstances connected with the question of their anniversary returns.

${ }^{37}$ Sill. xxxv., p. 363. Perhaps a part of the same cluster or ring was seen by Benzenberg, in 1798, on the 6th of December. The uncertainty concerning the convergent point prevents us from deciding. If this point were common, a period of forty years would give a relative velocity of about 11 geographical miles per second. 
TABLE II.

Convergent Point of the August Meteors.

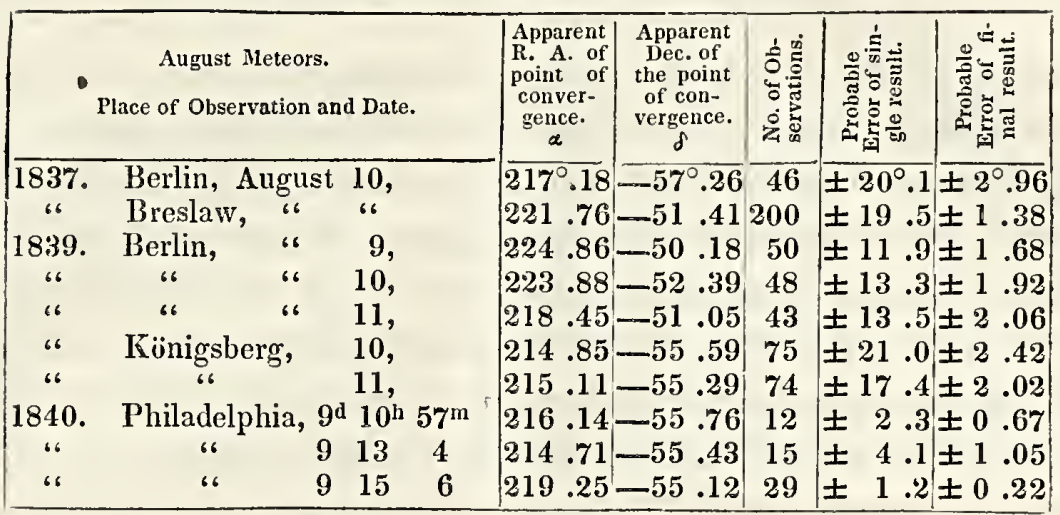

TABLE III.

Convergent Point of the November Meteors.

\begin{tabular}{|c|c|c|c|c|c|}
\hline & $\begin{array}{c}\text { Date. } \\
\text { Mean Time } \\
\text { Philadelphia }\end{array}$ & & $\begin{array}{c}\text { R. A. of } \\
\text { Convergent } \\
\text { Point. } \\
a\end{array}$ & $\begin{array}{c}\text { South Dec. of } \\
\text { Convergent } \\
\text { Point. } \\
\delta\end{array}$ & Observer and Station. \\
\hline \multirow[t]{9}{*}{1833} & November, & $12^{\mathrm{d}} .750$ & $333^{\circ} .0$ & $-17^{\circ} .0$ & Strong, Buffalo. \\
\hline & 6 & .701 & 328.0 & 15.0 & Merrick, Middletown, Ct. ${ }^{38}$ \\
\hline & :6 & .743 & 328.3 & 23.5 & “6 \\
\hline & 66 & .748 & 330.0 & 20.0 & Olmsted, New Haven.' \\
\hline & “6 & .745 & 329.0 & 21.7 & Riddell, Worthington, Ohio. \\
\hline & “ & .774 & 331.0 & 21.5 & 66 \\
\hline & 6 & .745 & 328.2 & 23.8 & Aiken, Emmettsburg, $\mathbf{M d}$. \\
\hline & 66 & .748 & 326.5 & 23.0 & Thompson, Mississippi. \\
\hline & 66 & .652 & 329.5 & uncertain & Parker, Gulf of Mexico. ${ }^{38}$ \\
\hline \multirow[t]{2}{*}{1834,} & 66 & .700 & 324.5 & 30.2 & Loomis, New Haven. ${ }^{39}$ \\
\hline & 66 & .700 & 330.0 & 20.0 & West Point. \\
\hline \multirow[t]{2}{*}{1835} & ، & 13.700 & 330.0 & 20.0 & Merrick, Amenia Academy, N. Y. \\
\hline & 6 & .700 & 332.7 & 20.7 & McCaffney, Mt. St. Mary's Col. Md. \\
\hline \multirow[t]{3}{*}{1836 , } & 66 & 12.631 & 326.7 & 30.1 & Dunster, Springvail, Maine. ${ }^{39}$ \\
\hline & ، & .631 & 325.0 & 25.0 & Olmsted, New Haven. ${ }^{39}$ \\
\hline & ، & .582 & 330.0 & 20.0 & Shæffer, New York city. \\
\hline \multirow[t]{5}{*}{1837 , } & "6 & .625 & 326.0 & 24.5 & Olmsted, New Haven. \\
\hline & 66 & .684 & 330.0 & 20.0 & Shæffer, New York city. \\
\hline & "6 & .666 & 329.8 & 20.9 & Barnard, New York city. \\
\hline & 66 & .667 & 330.0 & 20.0 & Obermeyer, Emmettsburg, $\mathrm{Pa}$. \\
\hline & 6 & .667 & 334.4 & 25.0 & Fitch, New Haven. \\
\hline \multirow[t]{3}{*}{1838 , } & "6 & .700 & $327 \cdot 0$ & 26.5 & Twining, Middleburg, Vt. \\
\hline & “ & .721 & 335.0 & 19.9 & Fitch, Gulf of Mexico. ${ }^{39}$ \\
\hline & 66 & 13.408 & 322.0 & -18.1 & Littrow, Vienna. \\
\hline \multicolumn{6}{|c|}{ Mean position of convergent point, $\begin{aligned} 1833-1838 a & =329^{\circ} .0 \\
\delta & =-22.0 \\
1833, \text { only, } a & =329.3 \\
\delta & =-20.7\end{aligned}$} \\
\hline
\end{tabular}




\section{§ III.-Of Periodical or Anniversary Displays of Meteors.}

The question of the periodicity, or the anniversary character of remarkable displays of shooting stars, was first started in 1833 and 1834, after the splendid spectacle of November 12th of that year, by Professors Olmsted and Twining, ${ }^{40}$ who had had "coincident ideas" on the subject. Three remarkable showers of these meteors have certainly occurred, in 1799, 1832, and 1833, at nearly the same point of the ecliptic, or earth's actual orbit; and either this coincidence must be accidental, or there is an unusual aggregation of these small bodies in the plane in which the earth is then found, which should lead us to expect other great displays to be witnessed in traversing the same part of our orbit. Whether any such have occurred since 1833 is a point that has been ably discussed by Bache, ${ }^{41}$. Olmsted, ${ }^{42}$ Twining, ${ }^{43}$ Lovering, ${ }^{44}$ Herrick, ${ }^{45}$ Forshey, ${ }^{46}$ and others in this country, and recently by many continental writers,

${ }^{40}$ Silliman, already quoted, vol. xxvi., p. 349, July, 1834. The following passage is explicit: "Hitherto we have reasoned from the known laws alone of the solar system; but the conclusion now forces itself upon every reader, that if these bodies had an orbit, they had also a period, and ought again to encounter the earth at some future time, or even to have encountered it in times past, in the same part of its orbit-that is, at the same time of the year. When, therefore, the startling confirmation of our theory springs up before us, that both the meteors of 1799 , seen by Humboldt at Cumana, and by Ellicott in the vicinity of the United States, and those of 1832 , seen at Mocha in Switzerland, and on the Atlantic, appeared at the same annual period with those of 1833, that is, on the 12th and 13th of November, (civil reckoning, we begin to feel as if further doubt were irrational."

${ }^{41}$ Sill., xxvii., p. 335, xxviii., p. 305, and xxix., p. 383. Prof. Bache considers the meteors on the anniversaries of November 12th-13th, 1834 and 1835, as presenting nothing extraordinary, either in numbers or direction of convergent point.

42 Silliman's Journal passim, since 1833. Prof. Olmsted thinks the anniversary returns have continued from 1831 to 1838 , and have since fallen off. See xxxv. p. 370 .

43 Sill. xxvii., p. 339. Prof. Twining considers the recurrence in November 12th, 1834, on a diminished scale as established, as also Nov. 13th, 1838. Sill, xxxv. p. 369.

${ }^{44}$ Idem. xxxv., p. 324. Prof. Lovering disbelieves in the anniversary returns of the November period since 1833, and ascribes the convergent point to the observer's motion.

${ }^{45}$ Idem., xxxviii. p. 377, and xl. p. 203.

${ }^{46}$ Mem. Am. Phil. Soc. 1840. 
Arago, ${ }^{47}$ Biot, ${ }^{48}$ Quetelet, ${ }^{49}$ Olbers, ${ }^{50}$ Boguslawski, ${ }^{51}$ Bessel, ${ }^{52}$ Littrow, ${ }^{53}$ and others. The earliest notice on this subject in Europe was by Arago, in 1835, after the November term of 1834 . The intimation on this subject by Olmsted, and its distinct announcement by Twining early in the summer of 1834, are matters of record, and have one year's priority to that of Arago, for the November meteors, and two years' priority to a similar announcement (since so happily verified) for the August period first made by Quetelet, in December, 1836. The last seven anniversaries of the 12th and 13th of November have been carefully watched in Philadelphia by Professor Bache and others, without rewarding them with the display of any unusual phenomenon, except, perhaps, that of the characteristic small deflection of the convergent point already described. At New Haven, and several other stations, however, an unusual number, and peculiar convergent point have been noticed; and in Europe, in 1836,1837 , and 1838 , the extraordinary phenomenon is said to have presented its anniversary return. Boguslawski reports its return in 1839, in moderate numbers. In 1840 , I believe, no unusual number was seen. The decision of the question, whether the theory of the annual periodicity of the November phenomenon must be considered as established by past experience, depends upon the sense in which the word periodicity is employed. If by this term we mean the recurrence of such remarkable and brilliant displays as those of 1799 and 1833, and, perhaps, of 1832 , it is clear that no return since 1833 has been witnessed. If, on the contrary, we restrict the term to the appearance of twenty or more of these bodies per hour, or twice the average number, I suppose that the years 1834-1839, inclusive, have presented such

47 Annuaire, \&c., 1836.

48 Sill., already quoted.

49 Catalogue, \&c.

50 Astr. Nachr. No. 372, p. 180 . Olbers considers the display witnessed by Kliiver, at Bremen, November 13th, 1838, as the " peculiar November phenomenon," such as occurred on the 11th, in 1799 , on the $12 \mathrm{th}$, in 1832 and 1833 , and since 1834 on the 13 th, always returning somewhat later. Those of the 12th November, 1838, though quite numerous, he rejects, as not moving in parallel paths, nor coming from the constellation Leo, as was the case with those of the 13 th.

51 Astr. Nachr. 391 and 412.

52 Bessel states the number of meteors seen November 13th, 1838, at the Königsberg Observatory, at one hundred per hour. Astr. Nachr., 371.

${ }^{53}$ Littrow reports the number, at same date, seen per hour at the Vienna Observatory, to be one hundred and sixty-seven, see Annalen, \&c., 1838, p. xvi., and ascribes the convergent point to the observer's motion in space.

VIII. - 2 A 
a phenomenon on the 12th or 13th of November, to some spectators, either in America or Europe. If we require, in addition to numbers, a parallelism of relative paths, and a common tendency towards a point within $8^{\circ}$ of the antipode of the observer's true direction, there is still no conclusive evidence of a failure of the phenomenon from 1832 to 1839 . Whether, however, their numbers or directions, except in 1832 and 1833, have in themselves any thing remarkable or extraordinary, or which extensive anniversary observations would not exhibit at other seasons of the year besides November and August, are questions on which a difference of opinion may naturally exist. The portions of earth's heliocentric longitude at which this peculiar November phenomenon, whether considered more or less extraordinary, has occurred, are given by Bessel in the paper above quoted, with a remark, that there are no grounds for a definitive conclusion either of the constancy of position of this point in the earth's orbit, or of a variation of it proportional to the time.

TABLE IV.

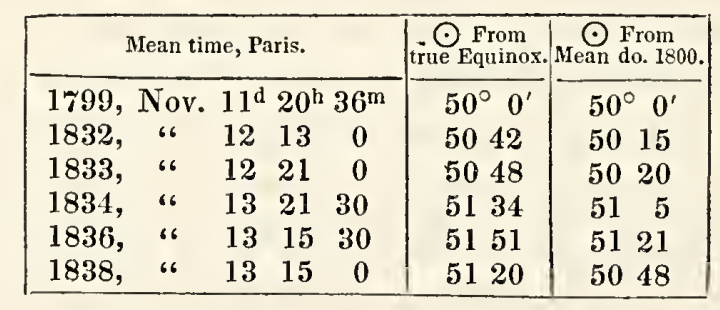

After all that has been written concerning the November-meteors, there is a possibility that the anniversary character of the meteor showers of 1799, 1832, and 1833 may have been accidental; that is to say, that the coincidence of the heliocentric longitude and radius vector of the three remarkable clusters that appeared at these respective dates, with those of the earth, may have been the natural consequence of the motions of three independent groups, having nothing else in common in their geometrical relations. In such a view of the case, the occasional appearance of an extraordinary number of these bodies, at certain positions on the earth's surface, on the 12th or 13th of November, since 1833, may have been the result rather of extraordinary assiduity in searching for them, than of any thing extraordinary in the distribution of these bodies in the system. The small deflection of the supposed convergent point of $8^{\circ}$ would then be re- 
garded as a possible error of observation, and a complete compensation of normal velocities would be considered as having taken place, and nothing would remain, either in their numbers or directions, but a promiscuous character; except, perhaps, a probable slowness of their true motions, making their relative tendency to the same point in the heavens more conspicuous.

There is another anniversary period about the tenth of August which may be termed Quetelet's period, from the circumstance of its first announcement by that celebrated philosopher, which is far better established than that of November, and presents phenomena more extraordinary, and more peculiar than have been witnessed at that of the latter, with the single exception of the great numbers in 1799, 1832, and 1833. This August phenomenon has been witnessed on more than half the anniversaries of this century. The group of bodies composing each display is more extensive, and the well determined mean normal velocity quite too great, to be explained on the supposition of their true directions and velocities being promiscuous in space. I shall here append Quetelet's table of the dates of remarkable showers of meteors, with a few additions, either more recent in occurrence, or since brought to notice, by Humboldt, Boguslawski, Erman, Jun., or others. A more extensive list has been prepared by Mr. Herrick, of New Haven, chiefly from his own researches, which it is hoped will soon be made public. The August and November meteors are most numerous from midnight to sunrise. This circumstance has been noticed by Prof. Olmsted ${ }^{54} \mathrm{Mr}$. Herrick, ${ }^{55}$ and others, and is ascribed, by the latter, to the position of the observer, whose geocentric direction then corresponds most nearly with that of his actual motion. Custodes, ${ }^{56}$ however, found the meteors of August 11th, 1834, most numerous before midnight. Mr. Herrick $^{57}$ has noticed, with respect to the meteoric abundance of Dec. 6th, 1798, and Dec. 7th, 1838, that the display was as great before as after midnight. This would lead us to suppose their relative velocity, of the latter, so great, as to have a character independent of the observer's motion. It has already been stated that the true velocity of the December flock must have been at least equal to that of the earth. Hence that we are led to conclude, from observation, that those meteors which for other reasons are supposed to have

${ }^{54}$ Sill. $x x x v$. p. 370 .

${ }^{56}$ Schu. Jahrb. 1838, p. 324, Note.
${ }^{55}$ Idem. xxxiii. p. 359, xxxvii. p. 333, and xxxix. p. 330. ${ }^{57}$ Idem. xxxv. p. 363. 
a slow true motion, give confirmation of the same, by appearing most abundant when the observer moves in front of the earth's centre-a circumstance that does occur with meteors moving more slowly in space.

Table V.

Catalogue of Remarkable Appearances of Shooting Stars.

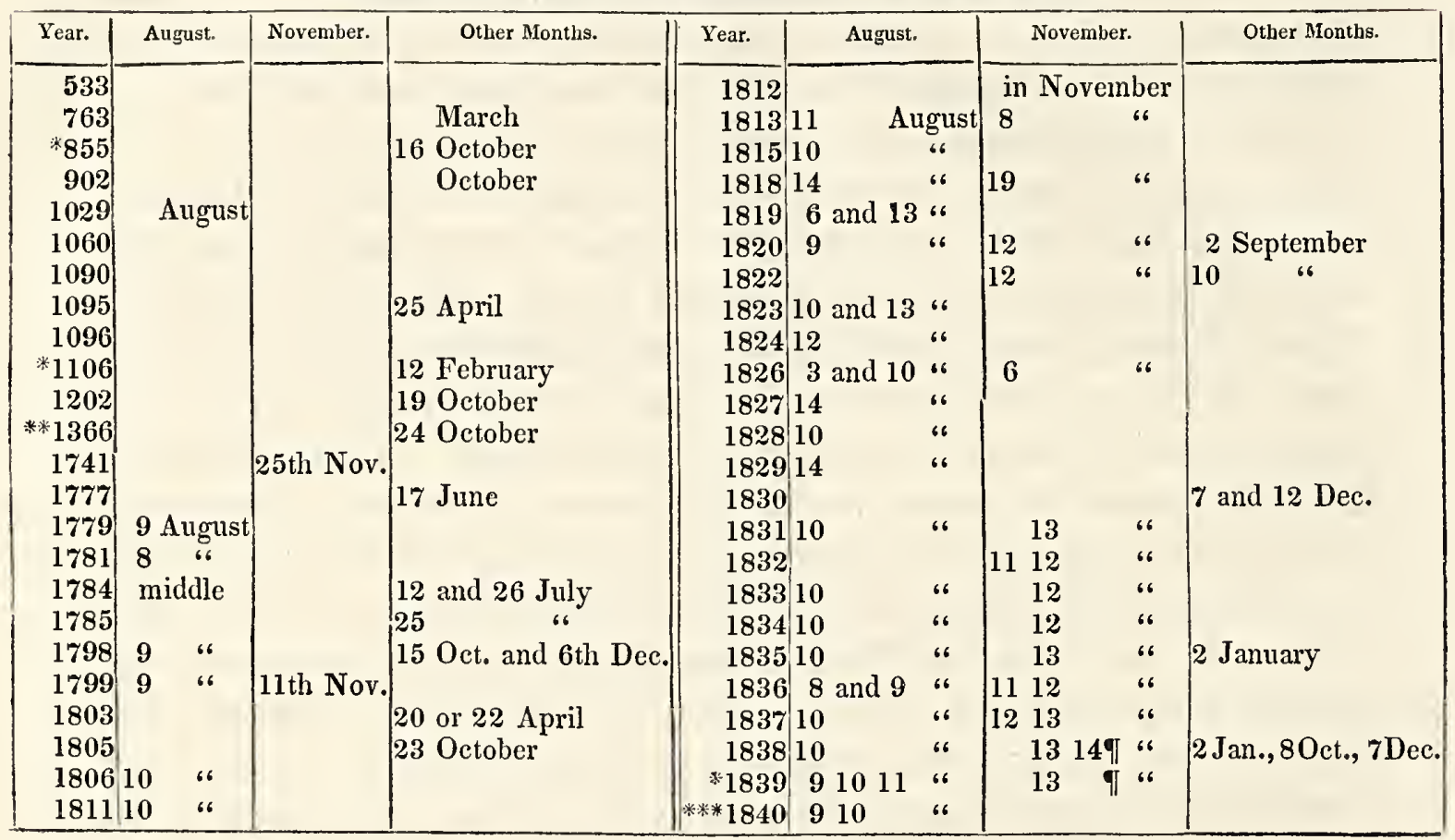

\$IV.-General Conclusions from the Facts stated in the Preceding SECTIONS.

The facts presented in the preceding sections suggest certain conclusions, as more or less plausible.

1. The existence of a convergent point, opposite to the observer's direction of motion is readily explained, by the known motion of the observer, and the fact, that such a point is observable, shows that there is a compensation of normal velocities, and hence a promiscuous character of the true directions and

58* Astr. Nachr. Nos. 391, and 412. 59 ** Ibid. No. 390. $\quad{ }^{80 * * *}$ Prof. Forshey's paper Mem. A. P. S. $1840 . \quad{ }^{61}$ q Sill. XXXV.p. 362. 
velocities of the meteors. The degree of deflection of single shooting stars from the theoretic convergent point, affords a classification of these meteors into sporadic and convergent, the former having a greater normal velocity than the latter, and hence, the effect of the other component of their relative velocity and direction being less manifest. This difference of velocities, naturally suggests a further classification of the orbits of these bodies, according to the cosmical theory, into very inferior asteroids, visible near their aphelion, and having small true velocities, and therefore conforming closely with the principal component of their relative directions, namely, the observer's tangential motion extended in an opposite direction. The sporadic meteors in contra-distinction from the others, may then be regarded as asteroids, superior to the convergent class, and, in many instances, superior to the earth. Then their true velocities being great, this component causes so great a deflection of their relative paths, that their relation to the other component is overlooked.

2. It is possible that the above classification may apply to the meteors of the 12th and 13th November, seen since 1833; since the supposed deflection of the convergent point of only $8^{\circ}$ or less, may be ascribed to the errors of observation.

3. The convergent point of the great shower of 1833 , which exhibited a similar deflection of about $8^{\circ}$, was much better ascertained, since telescopes were directed towards it, ${ }^{62}$ and its height was measured with a quadrant. ${ }^{63}$ This doubtless presents an exception to the last remark, in which case we must regard it as an extraordinary group, or cluster, or flock of these bodies, having a common, small normal velocity, and according to Twining's computations, a great relative velocity, nearly the same as that of the observer taken with an opposite sign. Such a cluster must then have had the other component or true velocity of the group very small, and hence its perihelion must have been near the sun. In fact, Prof. Olmsted's convergent point, and Twining's relative velocity, taken as data, would give an orbit coming nearly or quite in contact with the sun, and presenting the singular circumstance of the ballistic curve, so called with respect to the sun, in the same manner, as projectiles from gunpowder, or other forces on the surface of the earth.

4. The groups composing the displays on the same anniversary, in 1799,

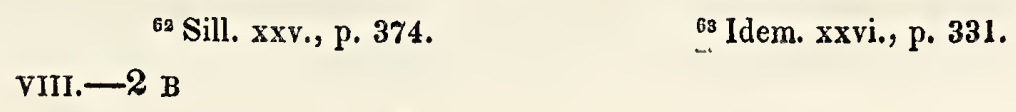


1832 , and 1833, may or may not have been parts of the same extensive nebula, or elliptic ring, or lens, having the sun in the focus of the orbit of each individual. On this point we are unable to decide, for want of knowledge of the deflection of the peculiar convergent points of the groups of 1799 , and $1832^{64}$. If the deflection of this convergent point had been known to be very different on these occasions, we should naturally pronounce them to have been different and independent groups. On the contrary, if this deflection had been known to be common, the probability of their being different portions of the same group, ring, or lens would be much increased, and would be a necessary conclusion, unless we admit, which I think is extremely improbable, a promiscuous character in their true directions and velocities, making a perfect compensation of normal velocities, and resolving the supposed deflection of $8^{\circ}$ into the errors of observation. In fact, to suppose a perfectly promiscuous character in the elements of motion, of such multitudes of bodies, and that after revolving around the sun for so long a term, they should by mere accident exhibit at the same period of the year, and same portion of space, three such brilliant displays as those of 1799,1832 , and 1833 , is to assign an effect without a cause.

5. Isolated groups, like that of December 7th, 1838, having no established anniversary or periodical character, but a great normal velocity, may be regarded as clusters of these small superior asteroids, having common elliptic elements, for whose approximate determination no other datum is wanting but the relative velocity of the group.

6. The display of the 10th of August, if witnessed only once, would lead to a conclusion similar to the preceding for December 7th, 1838. But when we consider that the same phenomenon is recorded for more than half the anniversaries of this century, and in every instance, when carefully observed, has exhibited the same true mean normal velocity and direction of motion, and the same node, and radius vector; it becomes far more worthy of the consideration of astronomers, and some law of distribution of the individuals composing these groups, and of the groups themselves, is required to explain these remarkable coincidences. Here all the peculiarities of periodical meteors are shown in their greatest perfection. The uniformity in their relative directions indicates

64 Olbers considers the character of parallelism of paths, of the bodies composing the displays of 1799,1832 , and 1833 , to have been common to the three displays. 
a similar uniformity of the other component, namely, their true velocity and direction, which, with that of the observer taken with a contrary sign, gives their relative velocity and direction as a resultant; for either there must be this uniformity, in their true velocities, or a compensation between the true velocities and directions-such as will produce a uniform true component in length and position. Now, the latter circumstance is extremely improbable. Its occasional occurrence might be considered accidental; but its repetition in nine hundred out of one thousand instances in one night, (the exceptions amounting to the tenth or twentieth part being readily accounted for,) indicates the prevalence of a general law, and we are thence compelled to suppose the true velocities and directions the same at the successive anniversaries. If this be admitted, then these successive groups, seen at yearly intervals, are moving, in each instance, in the same part of the same orbit. This identity of orbits is thus established. It will be seen in the sequel that there are only three independent variable elliptic elements of a meteor seen in proximity with the observer. These are the inclination $i$, the mean daily motion $n$, and the angle of eccentricity $\phi$; the three remaining elements (the ascending node $\Omega$, perihelion $\pi$, and epoch $H$, ) being known functions of the first three, and of the meteors' given position in the system. Now we have already arrived, by induction, at the conclusion that the true velocity $g$ is common in quantity and direction. An induction precisely similar, but having less force on account of the greater number of variables, and consequently of possible combinations, compells us to admit that these three elements, $i, n$, and $\phi$, are common to the successive convergent meteors. For though the same value of $g$ in quantity and direction might sometimes recur on the principle of a compensation of the discrepancies of $i$, $n$, and $\phi$, yet the uniformity of such recurrence (with only such exceptions as are referable to another law known to prevail) points to a uniformity of cause to be found only in the identity (or perfect similarity) of the successive combinations of $i, n$, and $\phi$, that is of the orbits of the successive individuals of the meteor group. Again, suppose, as in Table II., this principle of similarity of orbits established in this way for the individuals composing the respective flocks seen at Berlin and Breslaw in 1837, and also for those seen at Berlin and Konigsberg in 1839 . Then, since the three dependent elements, $\Omega, \pi$, and $H$, have no new feature in 1839 , the other three elements, $i, n$, and $\phi$, must be supposed to be common to the two phenomena of 1837 and 1839 , or we 
must suppose a compensation of their discrepancies throughout the two displays, an event the more improbable as the aggregate of the concurrent events of 1837 and 1839 is increased. These events amount in all to five hundred and thirty-six on these two occasions. When we add to these fifty-six concurrent results in 1840, and recollect that the number of events, confirmatory in their general character, and amounting to some thousands, as recorded in the observations at various stations in Europe and America in the years 18361840, about the 10th of August, the only legitimate conclusion is that these anniversary flocks of shooting stars are moving in the same part of the same orbit, round some central body. I have already shown that this central body can be no other than the sun. As there is no good reason for the contrary, I am led to extend the same general conclusion to the respective August anniversaries enumerated in Table V., and to suppose the tenth or twentieth part of the single meteors seen on those occasions which were unconformable, to have composed the ordinary promiscuous or sporadic meteors seen throughout the year. In such a case, these periodical phenomena, as Prof. Erman ${ }^{65}$ remarks, can only be explained by one of two hypotheses-either that the successive displays are parts of the same group or cluster, with a half yearly or yearly period, or that there exists in the particular part of the system which has for its radius vector 1.013, and its heliocentric longitude $137^{\circ}-$ $139^{\circ}$, without any latitude, a continuous succession of these bodies at all seasons of the year, moving always in the same orbit, though only encountered annually by the earth. It is obvious that the latter supposition of continuity of succession of these moving bodies at the same point of space, and in the same direction, requires the farther hypothesis of their forming a part of a conic-sectional ring, or lens, having the sun in its focus. In this case the anniversary display must continue to be witnessed while the radius vector, and anomaly of the node, remain the same, or while their periodical fluctuations are less than the breadth of the ring. When they exceed this breadth, the phenomenon ceases to recur, till, in the course of time, the node returns to the same anomaly and corresponding radius vector.

7. The remaining remarkable showers of meteors in the catalogue, may be accounted for by supposing the earth, at the successive dates, to have encoun.

${ }^{65}$ Astr. Nachr., Nos. 385 and 390. 
tered isolated nebulæ, or swarms or clusters of these asteroids. Perhaps the group, ring, or lens, which now furnishes the August or November displays, may have undergone, in the position of its plane, and line of apsides, such variations as to embrace a part of these remarkable epochs. On the hypothesis of a single cluster, with a yearly or half yearly period, this event would not be improbable. On the hypothesis of a ring, a change of plane, to a great extent, could hardly be considered probable. It seems more natural, however, since the relative direction of these asteroids in ancient displays, is unknown, in the absence of proof of identity with the groups of recent appearance, to regard the ancient displays of Quetelet's catalogue as isolated clusters, whose meeting with the earth was purely accidental; that is to say, was regulated by a law of the distribution of these single bodies, and their groups, in spacewhich must always remain unknown for want of data for its determination.

§ V.-Of the Respective Peausibilities of the Hypothesis of a single Cluster, with a half yearly or yearly Period, and that of a continuous Ring, for the periodical Meteors of August and November.

In order to judge of the plausibility of the theory of a single cluster and that of a continuous ring for the August and November asteroids, I here subjoin the results of a computation based upon these respective hypotheses; using, in connexion with them, the most plausible values of $\gamma, \alpha$, and $\delta$, derived from the preceding tables. These values are not sufficiently exact to furnish the particular elements of the orbits of these meteors. They serve, however, to show, by specific examples, that the general conclusions already announced concerning the character of some of their elements, the perihelion distance and semiaxis major, for instance, have not been drawn at random. The results would also be modified by the application of the effect of the earth's distur: bance, and of the resistance of its atmosphere.

$$
\text { vill. }-2 \mathrm{c}
$$


Table VI.

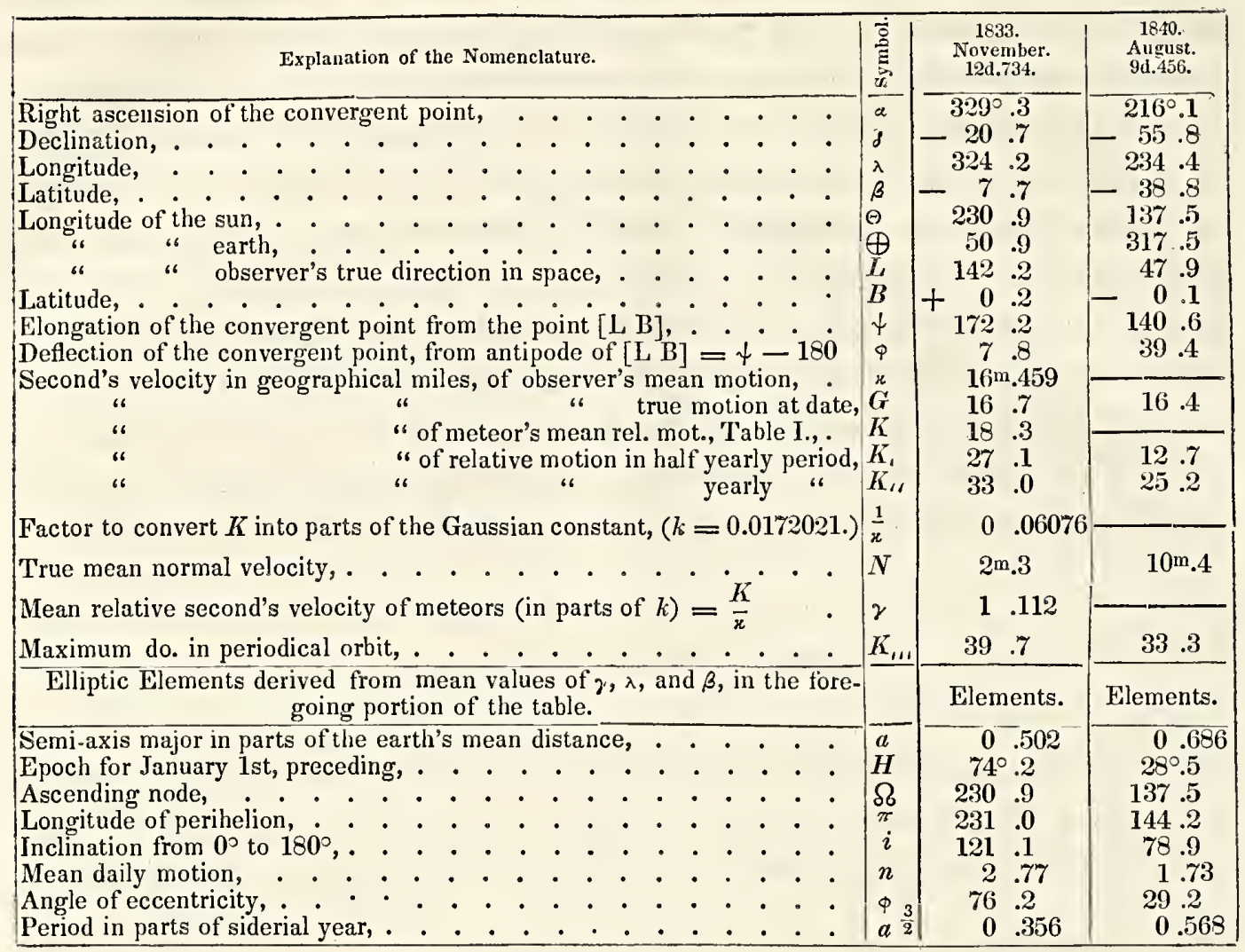

From this table it appears that a half yearly period, as originally proposed by Mr. Olmsted, or a yearly period as since maintained by Olmsted and Boguslawski, for the November meteors, or in other words, the anniversary return of a single cluster of these asteroids, cannot be reconciled with Twining's computed value of their relative velocity, nor with the mean result in Quetelet's table of these velocities, nor with Olbers' remark concerning Boguslawski's computations of the relative velocity of the meteors of November 13th, 1836 . Hence, notwithstanding the able arguments adduced by Boguslawski, in favour of the hypothesis of a single cluster, with annual returns for these meteors, the above objection seems insurmountable, and we are compelled to resort to one of the two hypotheses already stated, namely, that of the earth's accidental coincidence in position, with remarkable clusters in 1799,1832 , and 1833, and of a promiscuous character of the motions and orbits of the individuals composing 
the occasional abundances seen since 1833, on the 12th-13th November; or else to adopt, with Erman, that of a more or less continuous ring or lens with slight variations of the length and position of the radius vector of the node, so as to have exhibited the magnificent spectacles occasionally recorded, and at other years only a moderate abundance, or even an ordinary number.

For the August asteroids, the supposition of a single cluster with half yearly period, gives a relative velocity rather too small, and a yearly period too great to conform precisely with the average value in Table I.; and a period of 0.57 year affords a better agreement; still the half yearly value is within the limits of the probable errors of the observed mean value, and accordingly, the supposition of a single cluster with half yearly period being possible, there is no need of resorting, with Prof. Erman, to that of a continuous ring, unless the a priori probability of the latter hypothesis, or other circumstances which have been overlooked in this inquiry, tend to give greater plausibility to the theory of the existence of the ring, than to that of the cluster of half yearly period.

\section{§ VI.-Of certain Analogies in the Solar System and Siderial Heavens,} TENDING to CONFIRM the PRECEdING Conclusions.

I have already alluded to certain analogies, which would lead us to expect a gradual aggregation of these or other cosmical bodies in the vicinity of the sun, a conclusion, also, authorized from the observed geometrical relations of these asteroids, when seen by an observer on the earth. A similar law of aggregation is known to prevail with respect to the primary planets of our system. The same remark applies to the distribution of the perihelia of secondaries, or satellites round their primaries. This law of distribution of the perihelia of the primary and secondary planets round their central bodies, admits of very simple numerical formulæ for its expression. Comets, from the great eccentricity of their orbits, afford, perhaps, a closer analogy with meteors. Though the law of distribution of the perihelia of comets is unknown, still an inspection of the catalogue of their elements shows that four fifths have their perihelia beneath that of the earth, and six tenths beneath that of Venus. 
These circumstances, and the known fact that, in the different systems, inferiority of size and mass is generally connected with inferiority of perihelion distance, afford a double analogy for concluding a priori that the perihelia of these minute asteroids are gradually condensed as the distance from the sun diminishes, although the law of their aggregation in space will doubtless remain always unknown for want of data for its determination. Since countless millions of these bodies are annually encountered in the small portion of planetary space with which the earth comes in contact, we are led to the inference that the number of the perihelia of these bodies inferior to that of Venus, or even Mercury, is inconceivably great. Indeed, this would be the case, if these bodies were there scattered as sparsely as in the regions traversed by the earth. But the analogies I have mentioned strengthen the probability that no such rarity prevails within the limits of Mercury's, or Venus' mean distance; and this conclusion once arrived at, a new analogy comes in for its support. I allude to the extremely interesting discussion between Bessel and Encke, in Schumacher's Astr. Nachr., Nos. 289, 305, and 310, in which the former objects to the hypothesis of a resisting medium, from the fact that its existence is indicated by no other phenomenon in nature. The reply of the latter is, that no other phenomenon in nature is capable of its indication. Encke's comet is only found to be resisted while within the distance of Venus. Now as Halley's comet never goes far within the limit at which this resistance is sensible, and Biela's comet never approaches this limit, the perihelion distance of the former being superior to Mercury, and that of the latter little inferior to that of the earth, neither of these bodies can afford a contradiction or confirmation. The planets Mercury and Venus, the one within this limit, and the other on its border, are too dense and massive, compared with Encke's comet, to enable us to detect such a resistance. It is with deference to the opinion of these distinguished authors that I venture to suggest the possibility of such an aggregation of these small asteroids within the mean distance of Mercury, as may produce a sensible effect in resisting the progress of a body so light as Encke's comet, which Sir John F. W. Herschel ${ }^{66}$ supposes to be incomparably

${ }^{66}$ Herschel's Astronomy, Chap. x. 303. 'The author remarks, " that the most unsubstantial clouds which float in the highest regions of our atmosphere, and seem at sunset to be drenched in light, and to glow throughout their whole depth as if by actual ignition, must be looked upon as dense and massive bodies, compared with the filmy and almost spiritual texture of a comet." 
more rare than the thinnest clouds that float in the upper regions of the atmosphere. Should future observations establish on a firmer basis the general facts contained in the preceding tables, and thus warrant the opinion, that these small masses are much more closely aggregated together in the vicinity of the sun, then the theory which I have proposed, and that of the resisting medium will mutually strengthen each other. It may be further remarked, that should this theory of shooting stars be found to explain all their known phenomena, and to explain some portion of the resistance encountered by Encke's comet, an answer would be furnished to some interesting inquiries, by Sir J. F. W. Herschel in his Astronomy, page 310th, note. "What is the law of density of the resisting medium which surrounds the sun? Is it at rest or in motion? If the latter, in what direction does it move? Circularly round the sun, or traversing space? If circularly in what plane?" To these it would be answered, that the resistance is owing in part, at least, to the comet's meeting in its course an immense number of these small masses, each of which is pursuing its own orbit in a conic section round the sun, disturbed by the other planets, and by the meteors in its vicinity. That the law of their aggregation is unknown for reasons already mentioned; but that we have reason to suppose that the individual bodies retain their relative position or configuration by the general law of gravity, and not by any such law as that which regulates the relative position of the particles of an elastic fluid, or liquid, usually understood by the word medium.

I have thus discussed one feature of the present theory, namely, the gradual aggregation of these bodies in approaching the sun. I shall now suggest another, derived from the distribution of matter in the siderial heavens, of which the planetary space forms a part. This portion of space is continually changing, if the solar system, as was supposed by Herschel, and proved since by Argelander, ${ }^{67}$ has a proper motion in space. According to the opinion of Laplace, new portions of matter formerly revolving round that central body, or group, whose sphere of activity was greatest, must, in consequence of the motion of our system in space, be continually falling within the sun's sphere of attraction, and forming comets, asteriods, or planets. It is only necessary to extend the theory a little farther to include the small shooting stars, aerolithes, or asteroids. Now we notice in the heavens that matter is not uniformly distributed,

${ }^{67}$ Astr. Nachr., No, 363.

VIII. - $2 \mathrm{D}$ 
but we see a constant tendency to condensation into nebulæ of particular shapes and degrees of density-into the milky way-into clusters more or less resolvable-into rings, round, or perspectively, or really flattened--into planetary discs, which, when turned edgewise towards us, might present, as is often found to be the case, a section of a lens. Now that which is seen in the immensity of space, with bodies concerning whose dimensions no conjecture can be formed, may also occur in miniature, with respect to groups of these small asteroids, either from common circumstances connected with their original projectile motion, or origin, or time and place of first falling within the sun's sphere of activity, owing to the sun's proper motion in space. Or, in case of a uniform distribution at any time in a particular portion of the system, their mutual attractions, and those of the other bodies of the system, together with physical changes from variations of temperature, may naturally tend to produce the gradual formation of clusters, or, possibly, of circular or flattened rings or lenses, either continuous or interrupted, the individuals of which continue to circulate round the sun for many years almost in the same planes. A single flock or cluster of these bodies might extend for many thousand, or, possibly, hundred thousand miles, and have a general resemblance, though not a perfect uniformity of their elliptic elements. I have mentioned a general resemblance in their elements. This is all that could be expected, for a perfect uniformity of elements would require the several bodies of the group to be bound together by some law of fixedness of relative position, like the single particles of a solid, which does not here prevail.

The principal points in this theory for which I have endeavoured to find known analogies are,

1. That the perihelia of the orbits of these meteors are gradually aggregated together as the distance from the sun diminishes.

2. That by far the greater part of these bodies never reach the earth's mean distance.

3. That while this general law of distribution prevails in the planetary space, portions of this space have, besides the average number conformable to this law, special clusters, groups, or flocks of great extent, possibly composing entire rings or lenses embracing the sun.

4. That the individuals composing these clusters have similar elements of elliptic motion, and continue to move round the sun in a plane which, for a considerable period, undergoes but little change in space. 
5. That in such a plane there may be an indefinite number of these irregular groups at various mean distances from the sun; or there may be a tendency to the formation of a continuous, or imperfect and interrupted ring, the ring itself being a conic section, probably an ellipse. The ring of Saturn furnishes such an analogy in a secondary system, and, according to Cassini ${ }^{63}$ and Laplace, most probably the zodiacal light in the solar system.

Of the degree of plausibility of these hypotheses, a priori, every one must judge from such analogies as have been pointed out, and others which naturally suggest themselves. It will readily appear that if we admit them as the basis of the theory of shooting stars, we may readily infer from them the necessity of all the phenomena which I have pointed out as deductions from the established facts and statistics of meteorology.

By the general prevalence of the first and second law we should expect that a great portion of the shooting stars seen on ordinary nights, being small planets or asteroids, having their perihelia inferior to Mercury, must, at the time of visibility, be generally near their aphelia, and have a small space velocity, and, moving in all directions, the mean relative direction must be nearly opposite the observer's true direction, and the actual velocity of any single meteor resolved in the plain normal to the observer's direction must be small, and must exhibit itself as a small discrepancy from the mean convergent point for the evening. 'This character must prevail throughout the year, independent of any clusters or flocks which the earth may fall in with in its orbital motion.

On the same principle of distribution of perihelia, supposing all varieties of eccentricity to occur, some of these bodies, having perihelia inferior to that of Mercury, must have their aphelia far superior to the earth, and, by their mean distance and period, must belong to the class of superior planets. This class of superior asteroids of great eccentricity, and other asteroids having perihelia superior to the former, must, even with moderate eccentricities, still be considered as superior planets; and have, at the earth's mean distance, a true velocity greater than that of the earth; and moving in all varieties of directions, though the observer's true motion in space serves to impress on them a relative tendency opposite to his own, still the velocity of the asteroid in the normal plane being very great, the deflection of its relative direction from the mean 
convergent point, namely, the antipode of the observer's true direction, is so great that its connexion therewith does not appear, and the meteor is pronounced to be unconformable, non-convergent, or sporadic.

Again, if the third and fourth laws are supposed to prevail, the earth must, besides encountering the usual number of inferior and superior asteroids, also, on certain occasions, traverse the planes abounding in flocks or clusters already described, at the time when these clusters are in the nodes of their orbit. Still these flocks or groups of asteroids are not necessarily seen, since they only become visible to us when, besides being near their nodes, and we in the plane of their orbit, their radius vector is also within a few hundred miles, or, relatively to the earth's mean distance, sensibly the same as that of the observer. The coincidence in point of time of these three separate events, with reference to any one group or cluster of asteroids of moderate extent, supposing these groups to be distributed either indiscriminately in the system, or, according to a similar law to that which prevails relative to isolated asteroids, is a compound event of extreme improbability. Indeed, the visibility of a portion of such a group is only so far more probable than that of any isolated asteroid, as the cubical contents of the group are greater than those of the earth. ${ }^{69}$ Still, if the number of groups is indefinitely great, the event must sometimes occur. And if we attribute to our meeting with such isolated clusters the remarkable showers in Quetelet's catalogue, the only elements wanting would be the dimensions of these clusters, and an estimate of the annual number of isolated meteors encountered by the earth, in order to estimate the frequency of the distribution of these clusters in the system, compared with that of the single asteroids. When this complex event actually occurs, (our falling in with such a cluster, the individuals of which have similar, though not perfectly identical elements of motion round the sun,) the mean velocity of the group, in a plane normal to the direction of the observer's motion, deflects the convergent point from the antipode of the observer's direction. On such an evening, therefore, there should be two convergent points, the one for the asteroids of the cluster proper, the other the same as on ordinary nights. The convergent point for the cluster having hitherto been regarded as the principal convergent point, the other, if there be any, has always been overlooked, and all the isolated asteroids have been termed unconformable or sporadic.

${ }^{69}$ This is not quite exact, but near enough in general terms. 
§ VII.-Notice of several Theories of Aerolites and shooting Stars.

Previous to the researches of Brandès and Benzenberg, the prevailing opinion, with some exceptions, was that the shooting stars and fire-balls were of atmospheric or volcanic origin. Halley, whose labours, after Newton, established the rank of comets as periodical primary bodies of our system, was also of the opinion that the solar system contains myriads of small bodies, moving round the sun in conic sections. Many distinguished astronomers had maintained the same opinion, and Chladni had entered into an extensive discussion on this subject. He supposed that these isolated bodies pursue their paths in orbits unknown to us, till, by their entrance into the earth's atmosphere, they become luminous by ignition, and either just penetrating the surface of the atmosphere, leave it to pursue their orbit round the sun, or, entering more deeply, take fire and explode, or fall to the earth in the character of aerolites.

In 1775 Dr. Olbers, ${ }^{70}$ in a lecture at the museum of Bremen, suggested the idea of the lunar origin of aerolites, inclining, at the same time, to a belief that they were the product of the earth's volcanoes. The hypothesis of their volcanic origin was thought to receive confirmation from the fall of a shower of aerolites at Sienna, eighteen hours after an eruption of Vesuvius. Mr. Hamilton $^{71}$ had remarked that "stones of the same nature, as far as the eye could judge," had been found on Mount Vesuvius. Dr. Olbers, having computed the direction and projectile force required to send these fire-balls from Mount Vesuvius to Sienna in eighteen hours, and having found it not greater than that which is requisite for the up-heaving of mountains, a phenomenon known to have occurred in the historical period, was strongly persuaded of the correctness of the volcanic hypothesis.

The appearance of Howard's ${ }^{72}$ celebrated work on the chemical constituents of these aerolites, in 1802, and of Brandès' and Benzenberg's Researches on the distance, velocity, and orbits of shooting stars, in the same year, threw new light on the subject. The former showed that the chemical constituents of the

70 Zach's Monatliche Correspondenz, vol. vii., p. 148.

${ }^{71}$ Philosophical Transactions for 1795.

72 Idem., 1802.

VIII. $-2 \mathrm{E}$ 
meteoric stones were the same wherever found, and different from any known terrestrial product. The latter work showed that shooting stars, whatever might be the character of their chemical constituents, bore a close resemblance to the fire-balls in their geometrical relations, and had a velocity and altitude as great, if not greater, than those of the fire-balls whose paths had been determined. Howard's researches set aside, at once, the theory of the atmospheric or volcanic origin of the fire-balls, since, on such grounds, it was impossible to account for their chemical constituents. Accordingly, Laplace, ${ }^{73}$ in a letter to Zach, announces his theory of the selenitic origin of the fire-balls, and especially of the shower of aerolites at Sienna. He had found, by computation, that a projectile force of six times that of gunpowder, in a lunar volcano, would suffice to throw these masses beyond the sphere of the moon's activity, and make them satellites of the earth. The aerolites might then be the products of the lunar volcanoes sent forth in such a direction, and with such a velocity as to have their perigee within the earth's atmosphere. This view of the subject was maintained to the last by Laplace, and is repeated at length in his Systeme du Monde, published in 1824. This theory would account for the similarity of their chemical constituents, and would throw new light on the subjects of the constituents of the lunar volcanoes.

Olbers, in 1803, in the February number of the M. C., again brings forward his theory of their lunar origin, suggested, but not made prominent, in 1795, and gives a statement of the projectile velocity required at the moon's surface, namely, about 7780 Paris feet per second, in order that these bodies should reach the earth. Our cannon balls have a velocity of 1800 to 2000 Paris feet per second, as stated in the Memoires de l'Academie, \&c., of Paris, for 1769, p. 247 , et seq. The moon is known to be highly volcanic, and to have little or no atmosphere, hence the plausibility of this supposition of the requisite volcanic force being about four or five times that of gunpowder. There was a conclusion, however, from the observations of Brandès and Benzenburg, in 1798 , namely, that one of the meteors observed in common actually moved upwards from the direction of the earth's surface, which could not be explained either by the theory of their selenitic or cosmical origin. This circumstance, and the slowness of the motion of some spent fire-balls, induced Chladni to 
waive his theory of the casmical origin of meteors, and to resort again to that of a terrestrial origin. It does not appear that much new light was thrown upon this subject previous to the resumption of corresponding observations by Brandès and others in 1823, and Quetelet and others in 1824. The three theories, cosmical, selenitic, and terrestrial, had each their advocates. The first failed to account for the upward motion of meteors, if not for the uniformity of chemical constituents. The second accounted for the chemical facts, but failed to explain the upward motion of a meteor, as well as their great relative velocity, computed by Brandès in 1798. The last accounted for the upward motion, but not for the relative velocity nor chemical constituents. The chemical objection could be partly removed by a resort to the earth's atmosphere for the origin of motion; which would also account, though rather unsatisfactorily, for their upward motion, but would still be inadequate to explain their observed relative velocity. ${ }^{44}$

As late as 1834, Berzelius, ${ }^{75}$ and also Benzenberg, express themselves decidedly in favour of the Olbersian or selenitic theory. In 1836, however, Olbers, the original proposer of the theory in 1795, being firmly convinced of the correctness of Brandès' estimate of the relative velocity of meteors, renounces his selenitic theory, and adopts the cosmical theory as the only one which is adequate to explain the established facts then before the public. Arago and Quetelet had previously done the same. Littrow, in 1838, and Bessel, in 1839, fall in with the others. Professor Erman, Jun., in Berlin, and Boguslawski, ${ }^{76}$ in Breslaw, in 1839 and 1840 , have extended their inquiries on the bearing of the facts known up to these dates upon the cosmical theory. It is to Bessel's paper, already quoted, that we are indebted for the removal of the principal ground of objection to the cosmical theory, namely, that of the ascent of meteors. He has there shown that every instance in which Brandès' computations gave an upward motion of a meteor may be made to indicate the reverse, by applying a correction of the observed points of beginning and end of the apparent path of a meteor not much exceeding the probable error of such

${ }^{74} \mathrm{Dr}$. Olbers accounts for the serpentine motion of meteors that approach near the earth's sur* face, by partial explosions, after the manner of rockets.

${ }^{75}$ Olbers' paper, Schumacher's Jahrbuch for 1837, p. 54.

${ }^{76}$ Astr. Nachr., 391 and 412. 
observations. The chemical objection is not very weighty, for we may as well suppose a uniformity of constituents in cosmical as in lunar substances.

The opinion of Arago is contained in the following extract from Quetelet: ${ }^{77}$ "Brandès avait soigneusement appelé l'attention sur l'apparition remarquable des météores qui se montrèrent pendant la nuit du 10 Août, 1823, mais il avait perdu de vue que le même phénomène s'était déjà présenté, à lui, à la même époque, en l'année 1799. La supposition d'un retour périodique pour ces sortes de phénomènes, ne pouvait guère naître du reste qu'en présence de faits plus émergiquement prononcés. Il fallait le magnifique spectacle que déploya le nuit du 11 au 12 Novembre, 1832, pour réveiller la curiosité des savants et pour rappeler le souvenir effacé du phénomène tout aussi extraordinaire du 11 au 12 Novembre, 1799. Le hasard, on pourrait dire, plutôt que des combinaisons scientifiques, amena à constater un fait qui assure désormais aux étoiles filantes un rang si important dans notre système planétaire. Ainsi se confirme de plus en plus, comme le remarquait M. Arago, l'existence d'un zone composée de millions de petits corps, dont les orbites rencontrent le plan de l'écliptique vers le point que la terre va occuper tous les ans du 11 au 13 Novembre. C'est un nouveau monde planétaire qui commence à se révéler à nous."

That of Olbers is translated from his paper in Schumacher's Jahrbuch for 1837, pp. 279 and 280. "Perhaps the phenomenon of 1799 and 1833 has not been renewed. It appears, nevertheless, that a great multitude of the small molecules that compose the shooting stars move round the sun in orbits that intersect the plane of the earth's orbit, especially between the 19th and 21st degree of Taurus. These orbits, very close to each other, and completely parallel, compose, as it were, a common path for myriads, nay millions of these very small asteroids, which, in intervals nearly common, perhaps of five or six years, finish their circuit round the sun. They also seem to be quite unequally portioned out in this common path, sometimes in thiok swarms, sometimes more widely separated. In 1799 and 1833, and, perhaps, in 1832, the earth passed through one of these thickest swarms. In 1831, 1834, and 1836, only isolated, though numerous meteoric asteroids were met. Perhaps there are many of these thick swarms moving in this common path; perhaps, also, the inhabitants of the earth must wait till 1867 , before they see this wonderful

$$
77 \text { Catalogue, \&c., p. } 4 .
$$


phenomenon again in all its splendour, as in 1799 and 1833 . In the interval, however, it is of the highest importance that philosophers of every country should give the most careful attention, on the well known days of November, to the current appearances of these periodical shooting stars, as they are properly called in contradistinction from those sporadic meteors which occur throughout the year."

The objections of Dr. Olbers to the theory of the zodiacal light as the origin of the November meteors, ascribed to Biot, but, in fact, first proposed by Professor Olmsted, and still I believe maintained, are stated in the 281st and $282 \mathrm{~d}$ pages of the same work. Dr. Olbers pronounces it impossible to explain the motions of the November meteors, whose relative velocity he states at from sixteen to twenty geographical miles per second, by the supposition of a direct orbit round the sun, such as theory ascribes to the zodiacal light. Moreover, the nodes of the sun's equator are not in the 20th degree of Taurus, but in the $20^{\circ}$ of Gemini.

I may here remark, in confirmation of Olbers' statement, that the data of Tables I. and III., as presented in Table VI., give to the November meteors an orbit inclined $121^{\circ} .1$ to the ecliptic, in other words retrograde.

The opinion of the younger Littrow ${ }^{78}$ based upon a year's observations of these bodies, is as follows:- "Shooting stars are most probably of cosmical origin, as is shown by their return at stated periods of the year, and at particular portions of the heavens, both of which seem to depend upon the motion of the earth."

"The dates of the 10th of August and 12th of November are properly considered as periods when a richer fall of shooting stars may be expected."

"The phenomena of these two dates are different from those of ordinary nights. While the former exhibit a certain regularity in the place of their appearance and their directions, the latter seem to wander without rule in all parts of the heavens."

"The shooting stars of August and November are also of a different nature, in as much as they appear in quite opposite parts of the heavens, and the former are seen going towards that part of the celestial sphere from which the 
earth is coming, while the latter seem to come from a part of the heavens towards which the earth is going."

"Accordingly, the universe may be considered as replete with bodies of a similar kind, revolving round the sun. In certain portions, also, there is a tendency to the formation of connected systems of these bodies.. Two of these systems seem to describe paths round the sun that lie near the portions of the earth's orbit which it passes through in August and November. The peculiarities of the August and November meteors above mentioned may, perhaps, be best explained by supposing the orbit of the former nearly perpendicular, and of the latter nearly parallel to the orbit of the earth."

The latter supposition of Mr. Littrow cannot be reconciled with the conclusions in Table VI., drawn from the data of Tables I. and III., which give a high inclination for the November meteors, as was also remarked by Olbers.

Bessel's ${ }^{79}$ opinion has already been referred to. It is stated as follows:"Far more weighty grounds than those for ordinary shooting stars are at hand, which render it probable that those of November have a cosmical origin."

Professor Erman's theory of a ring for the August and November asteroids, and Professor Boguslawski's theory of a yearly period for the cluster of the latter, have been already alluded to.

The telescopic appearance of the meteors of the 9th and 10th of August, 1839, has been carefully described by the late Mr. E. P. Mason. And the opinion of that nice observer and zealous astronomer is contained in a manuscript not yet printed, and which is here offered to the public, in compliance with a request of the author made to me a few weeks before his decease.

"The nights of the 9th and 10th of August, 1839, are the evenings of the alleged recurrence of the August shower of meteors. They fell in extraordinary numbers, and of very uncommon brilliancy, during both nights. I have never seen nor heard of any telescopic observations of these bodies, and therefore take this occasion to offer my own on these evenings, as the statement would be too brief for a separate article, and I shall probably have no better

${ }^{79} \Lambda$ str. Nachr. 381, p. 349. "Es sind zwar Gründe vorhanden, welche den cosmischen Ursprung der November-Sternschnuppen, selbst vorzugsweise vor den gewöhnlichen, wahrseheinlich machen." 
opportunity of making it. Although it has no relation to the subject of nebulæ, which I was then observing, (unless we suppose these bodies to be the remnants of an original nebulous structure of our own system,) yet the subject of meteors is now attracting so much attention in Europe as to render the early publication of this notice not unimportant.

"During four or five evenings in the vicinity of August 9th, between twenty and thirty meteors passed the field of view. About twenty of these occurred on the 9th and 10th, during which nights I had the field almost constantly under my eye, until three or four o'clock in the morning. Their apparent brightness and velocity, as magnified by the whole power of the telescope, were, on the average, about the same, or rather less than that of those seen by the naked eye, (which latter class, to avoid repetition of the phrase, I will call the ordinary meteors.) They were of a very sensible size, more so than that of ordinary meteors of the same absolute brightness. On the average they were about half or one-third the diameter of Jupiter, and none were as large as that body. Their outline, however, was somewhat indefinite, like a star out of focus. In short, if such objects as the planetary nebulæ H. IV. 16 and 18, (which, and others of that class, had been observed a few evenings before,) could pass a field of view of between $30^{\circ}$ and $40^{\circ}$ of apparent diameter in about $0^{s} .2$ or $0^{s} .3$, I conceive they would exhibit, in every respect, all that could be gathered from so few of these objects during their brief intervals of transit. One only of the number appeared star-like, and of the twelfth magnitude. Their directions were so various that any judgment of their general tendency was relinquished.

"It is believed that these facts are not merely idly curious. We are enabled to gather from them the same information concerning the comparative remoteness of these telescopic meteors, that we already have of the relative distance of telescopic stars to those usually visible; for the chances were very great against the passage of a single ordinary meteor, during either night, across the minute space of sky actually occupied by the field of view. The appearance of so many of the telescopic within this space proved them to be vastly more numerous: and they were proportionally fainter; because invisible to the naked eye, and because the whole light of so large a telescope was unable to magnify them into an equality even with those seen by unassisted vision. Now, in these two particulars, great increase of number, and proportionate fee. 
bleness of individual light, consists all our knowledge of what remoteness to assign the telescopic fixed stars, and still farther on, the crowded hosts of the milky way, upon the scale of distance of which the nearest fixed star is the unit. The testimony is even far stronger in the case of the telescopic meteors; for the proportionate minuteness of their actual unmagnified velocity confirms, in the highest degree, what seems otherwise sufficiently evident, that we must allow them to have been many times farther off than those of ordinary occurrence. Why may we not gage the strata of meteors or meteoric matter, at the time of an expected shower, with that kind and degree of certainty which attends Sir William Herschel's gages of our sidereal system and milky way?

"Unless there is reason for a great difference between the absolute velocities of the more distant and the nearer of these bodies, the telescopic meteors which were seen on the above evenings could not have been much less than eighty times as far above the earth as those seen by the naked eye, which (according to the observations of Brandès and Benzenberg) probably darted most thickly at a height of fifty or sixty miles. This latter quantity, multiplied by eighty, or the magnifying power of the telescope, indicates a probable elevation of at least four thousand miles. At this vast height, if the atmosphere exist at all, it must be in a state inconceivably rare, rivalling the supposed resisting medium in its tenuity. It will at once be seen that telescopic observations, of the nature of those made with the fourteen feet reflector, have a peculiar bearing on the cause of the ignition of meteors, and, perhaps, on inquiries connected with the extent of the earth's atmosphere, and with the resisting medium. If carried out with energy, many of the misty theories concerning the nature and constitution of meteors will probably melt away, and we may have at least the comfort of compelling speculation to the effort of reinvention. A nebulous or gaseous constitution seems to be indicated by the observations, as far as they have a bearing on this point.

"I have seen (I believe in the London Times) a communication from Sir James South, the celebrated English astronomer, in which, after expressing great gratification at the recurrence of the annual shower on this same occasion, he remarks that he endeavoured to bring a hand telescope to bear upon the brightest of these objects, as they successively flashed, but without success, although 'a tolerably quick shot' in this kind of observation. This is the only attempt at telescopic examination of which I am aware." 
In the Journal of the Franklin Institute ${ }^{80}$ a letter addressed to Mr. Espy by me is published, describing a singular telescopic appearance noticed while watching for the emersion ${ }^{81}$ of $\mu$ Ceti from the dark limb of the moon, at forty minutes past twelve, on the night of the 7th of August, 1833, with a five feet Dollond, day eye-piece, power 30. The same event was seen by Mr. William H. C. Riggs, with a three and a half feet Dollond, and also by his assistant, Mr. Black, with the naked eye. Having observed the moon frequently for many years with a telescope, in the city of Philadelphia, and never witnessed any thing of the kind before nor since, I am inclined to ascribe the phenomenon to a cluster of small meteors. At the time, I supposed the bodies seen to be cinders from some neighbouring chimney. And it was not till after the great display of November 12th of the same year that my attention was called to this appearance, and its date identified by the occultation. The small bodies seen in the telescope traversing the moon's dise were semi-opake when on the disc, and had a phosphorescent appearance; their discs were not more than $20^{\prime \prime}$ in diameter. They appeared to move downwards, all in perfectly parallel directions. Sometimes several of them were seen traversing the moon's disc at once. The time of passing through the field was, perhaps, $0^{\text {s. }} 15$, as nearly as could be estimated. The downward motion observed at the time is consistent with the tendency towards the convergent point for the August meteors. The number seen could not have been less than fifty per minute in the field of view of the telescope, which was about a degree. The phenomenon lasted half an hour at least before the emersion; how long it continued afterwards I do not know, as no farther observations were made.

It must not, however, be forgotten that Professors Olmsted and Twining, who, early in 1834, had "coincident ideas on the subject," were the first to suggest the theory of annual periodicity of the November meteors, and to revive the cosmical theory in connexion therewith. In their early efforts at forming a theory, some opinions were advanced that have since been found untenable. Of this class may be mentioned the supposed parallax of the radiant point by the former giving for it a distance of about two thousand two hundred miles, and that of a parallax in declination by the latter. This last conclusion, based chiefly on the observations of Captain Parker in the Gulf of Mexico, in 1833, is, perhaps, set aside by the more recent and careful observations of Mr. Fitch,

${ }^{80}$ Vol. xV., p. 234 .

${ }^{81}$ Erroneously printed immersion of $m$ Ceti in that Journal.

VIII. $-2 \mathrm{G}$ 
in the same gulf, in 1838. Another opinion of Professor Olmsted, that the radiant body was relatively at rest with respect to the observer, is at variance with other known phenomena, since, according to the data of Section I., the relative velocity of the separate asteroids in space was not much inferior to that of the observer. Also, the radiant body itself, I suppose, must be given up, as no such stationary position of a gravitating body is possible, a relative motion being requisite to give sufficient centrifugal force to the body to prevent it from falling at once to the earth. Moreover, it was remarked by Professor Twining, and is now generally admitted, that the radiant and convergent points are the perspective vanishing points of lines nearly parallel. Professor Olmsted first adopted the half yearly period ${ }^{82}$ of a single cluster, to explain the anniversary phenomena of 1799,1832 , and 1833 . This he afterwards relinquishes for a yearly ${ }^{83}$ period. I have shown that neither the half yearly nor yearly period, with single cluster, will correspond well with the table of relative velocities, nor with that of the serpentine meteor as determined by Professor Twining. In reviewing Professor Olmsted's hypothesis respecting the observer's and radiant body's equal velocities and parallel directions, Mr. Espy ${ }^{84}$ intimates that such a coincidence is not possible in a perihelion or aphelion, but is possible in some part of the orbit of such a body. The following view of this question, differing from both, is derived from the analytical expressions for the tangential directions and velocities of any two bodies moving in orbits round the sun:-

$$
g=\sqrt{ }\left(\frac{2}{r}-\frac{1}{a}\right)=\frac{p}{r \sin u}
$$

(1)

$$
G=\sqrt{ }\left(\frac{2}{R}-\frac{1}{A}\right)=\frac{P}{R \sin U}
$$

In which

$$
\begin{aligned}
& a=\text { asteroids semiaxis major, } \\
& g=\quad \text { " } \quad \text { linear tangential velocity, } \\
& r=\quad \text { radius vector, } \\
& u=\quad \text { angle between radius vector and tangent to orbit, } \\
& p=\quad \text { semiparameter. }
\end{aligned}
$$

${ }^{82}$ Sill., vol. xxix., p. $378 . \quad \quad 83$ Idem., vol. xxxi., p. 393.

${ }_{84}$ Journal of the Franklin Institute, vol. xv., p. 10, note. 
The capital letters denoting the same quantities for the earth. Now, as Professor Olmsted supposes the velocities common, and the radii vectores are known to be common to terms of the order of the earth's radius divided by its radius vector, the semiaxes majores, $a$ and $A$, must be common, as well as the periods to the terms of the same order. Again, since $u$ and $U$ are supposed to be common in quantity and position, the parameters and consequent eccentricities, and also the planes of orbit must be common, that is to say, the two bodies must belong to the same system, and must have the ordinary secondary relation of primary and satellite, or they would immediately fall together. It appears, then, that such a coincidence as that which these authors refer to, is not possible in any part of the orbits of two bodies having respectively a half yearly and yearly period.

The mean angular second's motion $n$ of a satellite round the earth, in seconds of space, at the distance of its own radius, $\rho=\sin \varpi$, abstracting perturbations and resistance of medium, is

$$
\begin{aligned}
n & =\frac{1}{86400} \cdot \frac{k}{\sin 1^{\prime \prime}} \cdot \frac{\sqrt{ }\left(m+m^{\prime}\right)}{\sin ^{\frac{2}{2}} \varpi}=257^{\prime \prime} .08, \\
\nu & =\frac{n}{60}=4.285 \text { geog. miles, } \\
\sqrt{2} \times \nu & =6.06 \text { geog. miles. }
\end{aligned}
$$

Where

$$
\begin{aligned}
k & =\text { Gauss' constant given above, } \\
m & =\text { the earth's mass in parts of the sun's, } \\
m^{\prime} & =\text { the satellites } \\
m & =0.0000028192, \\
m^{\prime} & =0 \\
\varpi & =8^{\prime \prime} .5776=\text { sun's horizontal parallax, } \\
\nu & =\text { mean second's velocity in geographical miles. }
\end{aligned}
$$

Whence we have, as already stated, about six geographical miles as the greatest seconds motion which a periodical satellite can have round the earth. Hence the necessity of the cosmical theory; for we cannot refer them, with Professor Olmsted, to a radiant body with a yearly period, keeping itself constantly near the earth; nor can we suppose them satellites of the earth-nor projectiles from the moon-nor from the earth's volcanoes-nor atmospheric scintillations; and there remains no other plausible source of motion but the sun's attraction. 
\$ VIII.-Investigation of the Formule for conputing the Elliptic Elements of an Asteroid from its observed Relative Velocity and DiRECTION.

Having stated the general principles by which this modification of the cosmical theory is deduced from known facts, I shall proceed to point out the method of computation by which the data in Table VI. are obtained.

For this purpose let the true motion of the meteors and observer in space, as well as their difference, or the relative motion of the meteors, be referred to the rectangular co-ordinates $X, Y, \& Z$, having their positive values respectively directed towards the vernal equinox, the summer solstice, and north pole of the ecliptic, and let these motions be referred respectively to the sun's mass, earth's mean solar second, mean distance, and mean linear velocity, ${ }^{85}$ as the units of mass, time, space, and velocity. Let $G_{\circ}, L_{\circ}$, and $B_{\circ}$ denote respectively the earth's centre's true velocity, and the longitude and (latitude $=0$ ) of the point $L_{\circ}$ of the ecliptic towards which the earth's centre is moving in a tangential direction. Then, by the usual formulæ for the transfer from rectangular to polar co-ordinates, we have

$$
\begin{aligned}
& X_{\circ}=G_{\circ} \cos L_{\circ} \cos B_{\circ} \\
& Y_{\circ}=G_{\circ} \sin L_{\circ} \cos B_{\circ} \\
& Z_{\circ}=G_{\circ} \sin B_{\circ}=0 .
\end{aligned}
$$

In the same manner we have for the true tangential motion of the meteors in their orbit towards the point $[l b]$ with the velocity $g$,

(5)

$$
\begin{aligned}
& z=g \cos l \cos b \\
& y=g \sin l \cos b \\
& z=g \sin b
\end{aligned}
$$

and for their relative motion towards the point $[\lambda \beta]$ with the velocity $\gamma$

(6)

$$
\begin{aligned}
& \xi=\gamma \cos \lambda \cos \beta \\
& \eta=\gamma \sin \lambda \cos \beta \\
& \zeta=\gamma \sin \beta .
\end{aligned}
$$

Also $(\phi)$ denoting the geographical latitude, (the eccentricity of the earth's meridian being neglected,) and making $(G)=365.2564 \times \sin \left(8^{\prime \prime} .5776\right), G_{1}=$ $(G) \cos (\phi), \mu$ the siderial time, $\infty$ the obliquity of the ecliptic, we have for the velocity of the observer's actual rotary motion referred to the same axes,

${ }_{25}^{85}$ This unit is $\frac{k}{86400}$, where $k$ is the Gaussian constant given above. 


$$
\begin{aligned}
& X_{1}=G_{1} \cos \left(\mu+90^{\circ}\right) \\
& Y_{1}=G_{1} \sin \left(\mu+90^{\circ}\right) \cos \omega \\
& Z_{1}=-G_{1} \sin \left(\mu+90^{\circ}\right) \sin \omega
\end{aligned}
$$

and

$$
\begin{aligned}
& X=G \cos L \cos B=X_{0}+X, \\
& Y=G \sin L \cos B=Y_{0}+Y_{1} \\
& Z=G \sin B \quad=\quad+Z,
\end{aligned}
$$

$$
x=g \cos l \cos b=G \cos L \cos B+\gamma \cos \lambda \cos \beta=X+\xi
$$

$$
\begin{aligned}
& y=g \sin l \cos b=G \sin L \cos B+\gamma \sin \lambda \cos \beta=Y+\eta \\
& z=g \sin b=G \sin B+\gamma \sin \beta=Z+\zeta
\end{aligned}
$$

If we add together the squares of the three equations (8) $\psi$ being the angle of elongation of the convergent point from the observer's true direction, we obtain

(9)

$$
\begin{aligned}
g^{2} & =G^{2}+\gamma^{2}+2 G \gamma[\cos B \cos \beta \cos (L-\lambda)+\sin B \sin \beta] \\
g^{2} & =G^{2}+\gamma^{2}+2 G \gamma \cos \psi \\
\cos \psi & =\cos B \cos \beta \cos (L-\lambda)+\sin B \sin \beta \\
\gamma & =-G \cos \psi \pm \gamma\left(g^{2}-G^{2} \sin ^{2} \psi\right)
\end{aligned}
$$

In Table VI. $\psi$ comes out in the second quadrant both for the August and November meteors. Hence both signs before the radical are possible, and the only geometrical limit which these equations furnish, in order that $g, G$, and $\gamma$ shall be positive and rational, is that of the true norrnal velocity $(G \sin \psi$ ) This being necessarily the minimum of the true velocities, and the minimum required by the condition that $\gamma$ must be rational, we have for its limit (10)

$$
g=G \sin \psi
$$

Marking with a negative sign at top the quantities that result from the use of the lower or negative sign before the radical in the last of (9) we have the following geometrical limits of the values of $g$ and $\gamma$ :

Maximum of $g=+\infty$

$$
\text { " } \quad \begin{array}{rlrl} 
& \bar{g} & =G \\
& & \gamma & =+\infty
\end{array}
$$

$$
\text { Minimum of } g=G \sin \psi
$$

$$
\begin{aligned}
& & \bar{g} & =G \sin \psi \\
& & \gamma & =-G \cos \psi \\
& & \bar{\gamma} & =0
\end{aligned}
$$

VIII. -2 H 
These limits are derived from the principles of the geometry of position, and have no reference to the limits of $g$ and $\gamma$, deduced from the laws of elliptic motion. The most general interpretation of the cosmical theory of these asteroids is to suppose them to be moving in conic sections, in which those of (11) are the only necessary limits. In fact, the sporadic meteors, and the clusters which have no known periodical character, may have for their orbits either of the three conic sections. The case is quite different for the meteors which appear at anniversary periods, and annually exhibit the same normal velocity, too great to be ascribed to the errors of observation, and too uniform at each appearance not to be the result of identity of elements. The first principle of inductive reasoning which leads us not to assign two causes for an event where one is sufficient, would also lead us not to require for a series of connected events the repeated exertion of a cause, where a primitive exertion of it, and the subsequent action of known laws, are sufficient to account for the succession.

Now if we suppose that groups of bodies which, at yearly intervals, present the same combination of elliptic elements, are moving in non-periodical curves, (the parabolas or hyperbolas,) we must suppose that the primitive cause, whatever it may be, which gave to the bodies of our system their original projectile motion is continually exerted afresh so as annually to present the appearance just described. This is quite unreasonable; and accordingly, while we admit that the three classes of conic sections are possible for the sporadic meteors, and isolated clusters of unknown period, we must restrict those of anniversary occurrence to the class of ellipses, or periodical orbits, unless the observed relative velocity in Table I., combined with the convergent points in Tables II. and III., should require the contrary. Now, in equation (1) the maximum value of $g^{2}$ in a periodical orbit is ( $a$ being less than $+\infty$ ) equal to $\frac{2}{r}$ or $\frac{2}{R}$, neglecting the small discrepancies, or computing $R$ for the position of the observer, or meteor, if its distance from the observer is known. Then we have for the maximum value of $\gamma$ in a periodical orbit

$$
\gamma=-G \cos \psi+\vee\left(\frac{2}{R}-G^{2} \sin ^{2} \psi\right)
$$

The values of $\gamma$ in Table VI., computed from this formulæ, are, when multiplied by $x$, to convert them into geographical miles per second, respectively 39.8 for the November meteors, 33.3 for those of August, and 12.0 miles for those of December. As these values for August and November exceed the 
greatest velocities in Table VI, both observation and theory, in their present state, require us to restrict the anniveraary meteors to elliptic orbits. Analogy, also, leads us, in the case of sporadic meteors and isolated clusters, to make the same restriction. The value of 12.0 , however, for the December meteors, is too small to include all the values of Table $I$. in a periodical orbit, unless observation should show that, on the occasion of a high angle of deflection of the convergent point, the value of $x y$ falls below the average in Table $I$. The limits of (11) and (12) give for elliptic or periodical orbits

(13)

$$
\begin{array}{rlrl}
\text { Maximum of } g & =\sqrt{ }\left(\frac{2}{R}-\overline{a<+\infty}\right) \\
\text { " } & \bar{g} & =G \\
\text { " } & \gamma & =-G \cos \psi+\sqrt{ }\left(\frac{2}{R}-G^{2} \sin ^{2} \psi\right) \\
\text { " } & \quad \bar{a} & =+\infty \\
& & =\frac{1}{\bar{R}-G^{2}}
\end{array}
$$

$$
\begin{array}{rlrl}
\text { Minimum of } & g & =G \sin \psi \\
\text { " } & & \bar{g}=G \sin \psi \\
\text { “ } & \bar{\gamma}=-G \cos \psi \\
\text { " } & \bar{a}=\frac{1}{\bar{R}-G^{2} \sin ^{2} \psi}
\end{array}
$$

The limiting value of $g^{2}$, or $\frac{2}{R}$, is adopted by Olbers ${ }^{86}$ and Professor Erman, Jun. ${ }^{87}$ for the orbits of these asteroids. Their reasons for the restriction are not, however, stated. Professor Erman, Jun., has overlooked the limits which have the negative sign over them, and has, therefore, too much restricted the limits of the elliptic elements of these anniversary asteroids, possible according to the principles of the geometry of position. This oversight pervades the results and conclusions throughout that interesting paper. Its effect is particularly manifest in the formulæ there given for computing the maximum motion of the convergent point in a finite period, which he makes about $0^{\circ} .1$ per hour in a retrograde direction. Now, by applying the proper limit to the reciprocal of $\gamma$, which Professor Erman makes a coefficient of this motion, (since the limit of $\bar{\gamma}$ is 0 , and that of $\frac{1}{\bar{\gamma}}$ is $+\infty$,) we may have, without any 
contradiction from the geometry of position, a motion of the convergent point in a finite period of time indefinitely great, instead of $0^{\circ} .1$. There are other variable elements omitted in Mr. Erman's formulæ for computing this motion, which will be referred to in the next section. It remains to point out the use of the formulæ of (8) in computing the elliptic elements from observed values of $\gamma, \lambda$, and $\beta$, and the known values of $X, Y$, and $Z$. These equations may assume the following well known forms:

(14)

$$
\begin{array}{rlrl}
\tan l & =\frac{Y+\gamma \sin \lambda \cos \beta}{X+\gamma \cos \lambda \cos \beta} & = & \frac{Y+\eta}{X+\xi} \\
\tan b & =\frac{Z+\gamma \sin \beta}{X+\gamma \cos \lambda \cos \beta} \cdot \cos l=\frac{Z+\zeta}{X+\xi} \cdot \cos l \\
& =\frac{Z+\gamma \sin \beta}{Y+\gamma \sin \lambda \cos \beta} \cdot \sin l & =\frac{Z+\zeta}{Y+\eta} \cdot \sin l \\
g & =\frac{X+\gamma \cos \lambda \cos \beta}{\cos l \cos b} & =\frac{X+\xi}{\cos l \cos b} \\
& =\frac{Y+\gamma \sin l \cos \beta}{\sin l \cos b} & =\frac{Y+\eta}{\sin l \cos b} \\
& =\frac{Z+\gamma \sin \beta}{\sin b} & =\frac{Z+\zeta}{\sin b}
\end{array}
$$

In making the computations, either of the formulæ (9) may be used as a check to test the accuracy of the computation. The subsidiary quantities, $G_{\circ}$, $L_{\circ}$, and $B_{\circ}=0$, are readily obtained thus:

$(15)$

$$
\begin{aligned}
\oplus & =\text { the earth's longitude } \\
\varepsilon & =\quad \text { " eccentricity } \\
\Theta & =\quad \text { sun's longitude }=\oplus+180^{\circ} \\
R_{\circ} & =\quad \text { earth's radius vector } \\
G_{\circ} \vee R_{\circ} & =\sqrt{ }\left(2-R_{\circ}\right) \\
\vee\left(1-\varepsilon^{2}\right) & =G_{\circ} R_{\circ} \sin \left(L_{\circ}-\oplus\right) \\
(\phi) & =39^{\circ} .95 \\
G_{\triangleleft} & =0.011644=365.2564 \times \sin \varpi \cos (\phi)
\end{aligned}
$$

Now the quantities $L$ and $B$ differ from $L_{\circ}$ and $\left(B_{\circ}=0\right)$ by less than a degree, and the deflection of the convergent point from the plane of the $L_{\circ}$ and $B_{\circ}$, or, in other words, from the ecliptic, being in a southerly direction, and also southerly with respect to the plane of $L$ and $B$, the true motion of the meteor must be southerly, and it must be tending towards its descending node. Also, the 
meteor's radius vector and heliocentric longitude being sensibly the same as the observer's, and within quantities of the order of the earth's mean distance divided by its semi-diameter, the same as those of the earth's centre, those of the latter may be employed in the computation with sufficient precision, and we shall have, denoting by $\Omega$ the heliocentric longitude of the point $[l b]$ and making $u=$ the angle of inclination of the meteor's tangential direction to its radius vector, reckoned in the plane of its orbit in the order of the actual motion,

(16)

$$
\begin{aligned}
\Omega & =\oplus+u \\
\Omega & =\oplus, \text { for northern }=\Theta \text { for southern convergent point } \\
\Omega & =\Omega-\oplus \quad=\Omega-\Theta \\
\cot i & =\cot b \sin (l-\Omega) \\
\cos u & =\cos b \cos (l-\oplus) \\
g r \sin u & =\vee p=\vee a \cos \phi \\
p & =\text { semiparameter } \\
a & =\frac{1}{\frac{2}{r}-g^{2}} \\
\sin \phi & =e=\operatorname{sine} \text { of angle of eccentricity. } \\
v & =\text { true anomaly } \\
E & =\text { eccentric do. } \\
M & =\text { mean do. } \\
e r \cos v & =p-r=a \text { cos } \bar{\phi}-r \\
r \sin v & =a \cos \phi \text { sin } E \\
M & =E-e \text { sin } E \\
\pi & =\oplus-v=\Omega-u-v \\
k & =\text { Gaussian constant }=0.0172021 \\
\omega & =206264.67=\text { radius in seconds } \\
n & =k \vee(1+\mu) a-\frac{z}{2}=a-\frac{3}{2}, \text { for } k=1, \text { and } \mu=0 . \\
T & =a^{\frac{2}{2}}=\text { periodic time in siderial years. } \\
t & =\text { interval since preceding new year } \\
H & =\pi+M-n t=\text { epoch for preceding new year. }
\end{aligned}
$$

The values of Table VI. were computed by formulæ (14), (15), and (16). The numerical values of the fundamental equations are here subjoined, as they may save the labour of fresh computation by others who may engage in similar VIII.-2 I 
inquiries. The numbers, i, 2, 3, below the letters, refer respectively to the group of meteors of 1833, November 12.734, 1840, August 9.456, and 1838, December 7.333 .

(17).

$$
\begin{aligned}
& x_{1}=-0.80025+0.80423 \times \gamma_{1} \\
& y_{1}=+0.62056-0.57905 \times \gamma_{1} \\
& z_{1}=+0.00336-0.13385 \times \gamma_{1} \\
& x_{2}=+0.66830-0.45439 \times \gamma_{3} \\
& y_{2}=+0.74084-0.63354 \times \gamma_{2} \\
& z_{2}=-0.00252-0.62622 \times \gamma_{3} \\
& x_{3}=-0.98371-0.45439 \times \gamma_{3} \\
& y_{3}=+0.27412-0.63354 \times \gamma_{3} \\
& z_{3}=-0.00445-0.62622 \times \gamma_{3} \\
& \gamma_{1}=+1.00337 \pm V\left[g_{1}^{2}-0.01874\right] \\
& \gamma_{2}=+0.77144 \pm V\left[g_{2}^{2}-0.40035\right] \\
& \gamma_{3}=-0.27612 \pm V\left[g_{3}^{2}-0.96660\right]
\end{aligned}
$$

§ IX.-OF the Variations of the RELative Velocity and Convergent PoInt.

The convergent point on ordinary nights, according to the observations of Mr. Herrick and Mr. Forshey, varies with the point which is opposite the observer's true direction. And if there is a similar tendency to compensation of the relative velocities, then the mean relative velocity of shooting stars varies with that of the observer. I have already referred to Professor Erman's formulæ for this variation, and have shown that the restrictions are too great, from an unnecessary limit of the value of the reciprocal of $\gamma$. Let us suppose that the observer, in his annual and rotary motion, falls in with a group of these bodies having nearly the same elements, and that he encounters the individuals at successive dates, $t, t^{\prime}, t^{\prime \prime}, \& c$. It is manifest that if all the other elements were common, the position of the plane of the orbit of the successive meteors must vary with the observer's change of position, so that the elements 
cannot be identical. The differences may be embraced in two classes, those which increase with the time, and those which arise from discrepancies of the elements. Denoting the former by $\Delta$, and the latter by $d$, and their joint effect by $\delta$, we have in the interval $\left(t^{\prime}-t\right)$,

(18)

$$
\begin{aligned}
& \delta \xi=\xi^{\prime}-\xi=\xi+\mathrm{d} x+\Delta(x-X) \\
& \delta \eta=\eta^{\prime}-\eta=\eta+\mathrm{d} y+\Delta(y-Y) \\
& \delta \zeta=\zeta^{\prime}-\zeta=\zeta+\mathrm{d} z+\Delta(z-Z)
\end{aligned}
$$

for the variations of $\xi, \eta$, and $\zeta$. Hence, in estimating the variations $\delta \gamma, \delta \lambda$, and $\delta \beta$, some allowance must be made for the quantities $\mathrm{d} \gamma, \mathrm{d} \lambda$, and $\mathrm{d} \beta$, arising from discrepancies of the true elements of the meteors seen at the dates $t, t^{\prime}, \& c$. Professor Erman's formulæ, on the contrary, proceed upon the presumption of $\mathrm{d} \xi=-\Delta X, \& \mathrm{c}$. Now, as $\mathrm{d} x,+\Delta x, \& c$. , cannot, even in the thickest flocks of meteors, vanish entirely their aggregate effect in a finite interval of a few hours, may be such as to preponderate over that of $\Delta(-X)$, and in this manner the variation $\delta \lambda$, in a few hours, may come out positive, as reported by Professor Forshey, August 9th, 1840. Also, on ordinary nights, if a convergent point is found to prevail, we should have

$$
\delta \xi=\xi^{\prime}-\xi=\xi+(\mathrm{d} x+\Delta x)-\Delta X=\delta x-\Delta X
$$

and so on for $\delta \eta$ and $\delta \zeta$ : now if during an interval $t^{\prime}-t$ of several nights we find by observation, with Mr. Fitch and Mr. Herrick, $\delta \lambda=\Delta(L+180$, $\delta \beta=-\Delta B=0$, we are led to the inference that $\delta \gamma=\Delta(-G)$, and $\mathrm{d} \xi=-$ $\Delta X, \& c$. And that a compensation has taken place among the true velocities and directions of the meteors seen near each date, so that the convergent point has corresponded, in position and variations, with those of the antipode of the observer's actual direction.

On the occasion of great displays like that of November, 1833, when telescopes were directed to the radiant point, and its altitude was measured with a sextant, it is probable that $\mathrm{d} x, \mathrm{~d} y$, and $\mathrm{d} z$ were very small; in such a case a precise measure of the position of this point in the heavens might possibly, by giving the value of $\delta \lambda$ and $\delta \beta$ in a finite interval, enable us to determine $\gamma$ from the terms $\mathrm{d} x, \mathrm{~d} y$, and $\mathrm{d} z$, of which it would be a function. I do not, however, think such a precision can ever be obtained.

There is another point of view in which the knowledge of the values of $\mathrm{d} x_{\mathrm{z}}$ 
$\mathrm{d} y$, and $\mathrm{d} z$, for variations of $\gamma, \lambda$, and $\beta$, may be useful, and that is in enabling us to estimate the probable errors of any system of elements for a group or cluster of these meteors derived from assumed values of $\gamma, \lambda$, and $\beta$. The formulæ for computing variations of elements for a change of radial positions and distances in an orbit, or of geocentric positions and distances in the heavens, are stated at length by writers on the laws of elliptic motion, Gauss, Littrow, Santini, and others. As I do not recollect to have met with similar expressions for the variations of tangential directions and velocities, or relative direcrections and velocities, I shall here point out the method employed in preparing the requisite formulæ.

In the formulæ (8), making $\gamma^{\prime}=\gamma \cos \beta, g^{\prime}=g^{\prime} \cos b$, and differentiating, we obtain, after making the requisite reductions,

$$
\begin{aligned}
\mathrm{d} g^{\prime} & =+\cos (\lambda-l) \cos \beta \cdot \mathrm{d} \gamma-\frac{\gamma^{\prime}}{\omega} \sin (\lambda-l) \cdot \mathrm{d} \lambda-\frac{\gamma^{\prime}}{\omega} \cos (\lambda-l) \tan \beta \cdot \mathrm{d} \beta \\
\mathrm{d} l & =\frac{\omega}{g^{\prime}} \sin (\lambda-l) \cos \beta \cdot \mathrm{d} \gamma+\frac{\gamma^{\prime}}{g^{\prime}} \cos (\lambda-l) \cdot \mathrm{d} \lambda-\frac{\gamma^{\prime}}{g^{\prime}} \sin (\lambda-l) \tan \beta \cdot \mathrm{d} \beta \\
\text { (20) } \mathrm{d} b & =\frac{\omega}{g^{\prime}} \cos ^{2} b[\tan \beta-\tan b \cos (\lambda-l)] \cos \beta \cdot \mathrm{d} \gamma \\
& +\frac{\gamma^{\prime}}{g^{\prime}} \cos b \sin b \sin (\lambda-l) \cdot \mathrm{d} \lambda \\
& +\frac{\gamma^{\prime}}{g^{\prime}} \cos ^{2} b[1+\tan b \tan \beta \cos (\lambda-l)] \cdot \mathrm{d} \beta
\end{aligned}
$$

Expressions equivalent to those given by the authors above quoted for the variations of positions and distances, as might be expected from the symmetrical form of the fundamental equations, from which they are derived. Also, as before, $\Omega=\pi+v+u$, denoting the longitude on the orbit of the point [ $l b]$ towards which the true motion of the asteroid is directed, and $\Omega-\Omega=\pi+v$ $+u-\Omega$, being the argument of latitude, the equations

$$
\begin{aligned}
\tan (l-\Omega) & =\cos i \tan (\pi+v+u-\Omega) \\
\sin b & =\sin i \sin (\pi+v+u-\Omega) \\
\tan b & =\sin i \tan (\pi+v+u-\Omega) \cos (l-\Omega) \\
& =\tan i \sin (l-\Omega) \\
\cos (\pi+v+u-\Omega) & =\cos b \cos (l-\Omega) \\
g^{\prime} & =g \cos b
\end{aligned}
$$

(21)

give by differentiation, substitution, and reduction, 


$$
\begin{aligned}
\mathrm{d} g^{\prime} & =\cos b \cdot \mathrm{d} g-\frac{g}{\omega} \sin b \sin (l-\Omega) \cdot \mathrm{d} i-\frac{g}{\omega} \sin b \sin i \cos (l-\Omega)[\mathrm{d} \pi+\mathrm{d} v+\mathrm{d} u-\mathrm{d} \Omega] \\
\mathrm{d} l & =-\mathrm{d} \Omega-\tan b \cos (l-\Omega) \cdot \mathrm{d} i+\frac{\cos i}{\cos ^{2} b}[\mathrm{~d} \pi+\mathrm{d} v+\mathrm{d} u-\mathrm{d} \Omega] \\
\mathrm{d} b & =\sin (l-\Omega) \cdot \mathrm{d} i+\sin i \cos (l-\Omega)[\mathrm{d} \pi+\mathrm{d} v+\mathrm{d} u-\mathrm{d} \Omega]
\end{aligned}
$$

Expressions which I do not recollect to have met with before. They differ from the formulæ given by Gauss, Theoria Motus, p. 49, and by Santini, Elementi di Astronomia, Vol. I., Cap. XVII., Prob. IX. and X., and by other writers on the theory of elliptic motion, in containing tangential directions and velocities instead of orbital positions and distances, and having in the bracket an additional variable, $u$, which does not enter into the expressions for the variations of the latter class, and which here introduces new relations between the remaining elements $\phi$ and $n$, of which it is a function.

In order to obtain $\mathrm{d} g^{\prime}, \mathrm{d} l$, and $\mathrm{d} b$, in terms of the variations only of the elements proper, we must substitute the values of $\mathrm{d} g, \mathrm{~d} v$, and $\mathrm{d} u$, in terms of those of the elements. From the Theoria Motus, p. 15, with small modifications, we have (23)

$$
\mathrm{d} v=\frac{a a}{r r} \cos \phi\left[\mathrm{d} H+t_{l} \mathrm{~d} n-\mathrm{d} \pi\right]+\frac{(2+e \cos v) \sin v}{\cos \phi} \cdot \mathrm{d} \phi
$$

$$
\mathrm{d} r=\frac{r}{a} \cdot \mathrm{d} a+\frac{a}{\omega} \tan \phi \sin v[\mathrm{~d} H+t, \mathrm{~d} n-\mathrm{d} \pi]-\frac{a}{\omega} \cos \phi \cos v \cdot \mathrm{d} \phi
$$

and from the equations

(24)

$$
n=a^{-\frac{3}{3}}
$$

$$
p=a \cos ^{2} \phi \cdot=g^{2} r^{2} \sin ^{2} u=\left(\frac{2}{r}-\frac{1}{a}\right) r^{2} \sin ^{2} u
$$

by means of differentiation, making, for conciseness, $N^{\prime}=\frac{r-a}{2 a-r}$, and putting for $\tan u$, its value $=\tan (\Omega-(\pi+v))$

(25)

$$
\begin{aligned}
& \mathrm{d} n=-\frac{3}{2} \cdot \frac{n}{a} \cdot \mathrm{d} a \\
& \mathrm{~d} g=\frac{1}{2 a a g} \cdot \mathrm{d} a-\frac{1}{r \frac{1}{r g}} \cdot \mathrm{d} r \\
& \mathrm{~d} u=\tan (\Omega-(\pi+v))\left[\frac{2}{3} N^{\prime} \cdot \frac{\mathrm{d} n}{n}+N^{\prime} \omega \cdot \frac{\mathrm{d} r}{r}-\tan \phi \cdot \mathrm{d} \phi\right]
\end{aligned}
$$

expressions which, substituted in (22), would, after reduction, furnish the general solution of the problem for all relations of an orbit to the ecliptic.

$$
\text { viII. } 2 \mathrm{~K}
$$


In the present case, the asteroid being near the observer, or (neglecting the small quantities already mentioned) near the earth's centre, and consequently near its node, recollecting that

(26)

$$
\begin{aligned}
\tan u & =\tan (\Omega-(\pi+v))=\tan (\Omega-\oplus)=\tan (\Omega-\Omega) \\
\sin (l-\Omega) & = \pm \sin (l-\oplus) \\
\cos (l-\Omega) & = \pm \cos (l-\oplus) \\
\tan b & =\tan i \sin (l-\Omega) \\
\mathrm{d} \Omega & =0 \\
\mathrm{~d} \pi+\mathrm{d} v & =\mathrm{d} \oplus=0
\end{aligned}
$$

and using the upper sign for a convergent point north of the ecliptic, and observing that $\mathrm{d} H$ may be neglected, since it could only be introduced into these formulæ by making part of the value of $\mathrm{d} v$, which, in this instance, disappears, and calling $N=\frac{2}{3} \cdot \frac{r-a}{2 a-r} \cdot \frac{1}{n}$, and substituting and reducing, there result for the case of a meteoric asteroid, the following expressions of the meteor's true cur tate velocity and direction, in terms of those of the three independent elements,

$$
\begin{aligned}
\mathrm{d} g^{\prime} & = \pm \frac{g^{\prime}}{\omega} \tan b \sin (l-\oplus) \mathrm{d} i+\frac{g^{\prime}}{\omega} N\left[\frac{r}{2(a-r)}-\tan ^{2} b\right] \cdot \mathrm{d} n+\frac{g^{\prime}}{\omega} \tan ^{3} b \tan \phi \cdot \mathrm{d} \Phi \\
\mathrm{d} l & =\mp \tan b \cos (l-\oplus) \cdot \mathrm{d} i+N \cdot \frac{\tan (l-\oplus)}{\cos ^{2} b} \cdot \mathrm{d} n-\frac{\tan (l-\oplus) \tan \phi}{\cos ^{2} b} \cdot \mathrm{d} \Phi \\
\mathrm{d} b & = \pm \sin (l-\oplus) \cdot \mathrm{d} i+N \tan b \cdot \mathrm{d} n-\tan b \tan \phi \cdot \mathrm{d} \phi
\end{aligned}
$$

More simple expressions for the variations of some of the elements may be derived from the equations (9) and from the expression

(28)

$$
\sqrt{ } a \cos \phi \sin i=g r \sin b=r Z+r \gamma \sin \beta
$$

thus respectively (29)

$$
\begin{aligned}
& \mathrm{d} n=-3 n^{\frac{1}{3}} \omega(\gamma+G \cos \psi) \cdot \mathrm{d} \gamma-3 n^{\frac{1}{3}} G \gamma^{\prime} \sin (L-\lambda) \cdot \mathrm{d} \lambda+3 n^{\frac{2}{3}} G \gamma^{\prime}[\cos (L-\lambda) \tan \beta-\sin B] \cdot \mathrm{d} \beta \\
& \mathrm{d} \phi=\cot \Phi \cot i \cdot \mathrm{d} i-\frac{\cot \phi}{3 n} \cdot \mathrm{d} n-\frac{r n^{\frac{1}{3}} \omega \sin \beta}{\sin i \sin \phi} \cdot \mathrm{d} \gamma-\frac{r n^{\frac{1}{3}} \gamma^{\prime}}{\sin i \sin \phi} \cdot \mathrm{d} \beta
\end{aligned}
$$

These expressions establish the principle stated in Section IV., No. 6, that the independent elements may be reduced to three. They would, if the data of Tables I., II., and III. possessed the requisite precision to warrant the presumption that the elements of Table VI. are approximations towards the real values, enable us to estimate the effect of the uncertainty of the assumed values of $\gamma, \lambda$, and $\beta$. At present this cannot be considered the case. It is worthy 
of remark, however, that by proceeding from the values of Table VI. for the November meteors, and making $\gamma, \lambda$, and $\beta$ vary within limits assigned to their probable errors by Professor Twining, while the general character of $i$ and $a$ remains the same, that is to say, retrograde or highly inclined, and inferior, the value of $\phi$ may approach nearly to $90^{\circ}$, and the perihelion distance, $\frac{p}{1+e}$, may approach the value of the sun's semi-diameter, or $\sin \left(16^{\prime} 1^{\prime \prime}\right.$.) This will readily be inferred from the largeness of the negative coefficient of $\mathrm{d} \gamma$ in the last equation of (29). This circumstance gives to the remarkable serpentine meteor of the great November display, as has been already stated, the character of an emanation from the sun's atmosphere. How far such a conclusion, founded on this single result, may be considered as plausible, must be left to others to decide. I will merely remark, that if the position of the convergent point had been such as to give this value of $\phi$, with $i$ small, and a consequent motion direct, this circumstance would be somewhat confirmatory of the nebular hypothesis of Laplace; since, if one class of small asteroids may with reason be supposed to have had their origin in the sun's atmosphere, analogy may authorize us to suppose a similar origin for the projectile motion of the larger asteroids and planets, in the gradual condensation of the nebulous portions of matter composing the system, the motion of rotation being converted into an orbital motion. The analogy fails, however, on account of the high inclination of the orbit of the meteor derived from the same data.

\section{NOTE.}

In Section I. of this paper I made mention of the observations of Professor Locke, published in 1834, in the "Cincinnati Daily Gazette." Those of the 8th and 10th of August, 1834, published on the 11th and 12th of August in the same year, are worthy of being reprinted from the files of that paper, as they show that, although the periodicity of the August meteors was first discovered by Quetelet in 1836, the position of their radiant and convergent points was first discovered, and pointed out with precision by an American, in 1834, as had been done the previous autumn by Professor Olmsted and others for the No. vember meteors.

Cincinnati Daily Gazette, August 11 th, 1834 .

"Mr. Editor, "METEORS.

"On the evening of August 8th I observed, in the course of two hours, thirty meteors or "shooting stars." As I could not have in view more than one-fourth of the visible heavens at once, there were probably one hundred and twenty meteors to be seen in that time. I do not mention this as any thing uncommon, but 
merely to draw the attention of astronomers to the subject. If they will mark the course of remarkable meteors upon the fixed stars, and note the time, we can obtain the parallax of some identical one, and thus ascertain its place in the regions of space. If observers at Dayton, Oxford, Lexington, Louisville, \&c., will join me, I will devote the hours from 6 to 10, and, in some cases, from 8 to 11, to observations of this kind.

"The following observations were made on the evening of the 8th:-

“1. $9 h .25 \mathrm{~m} .30 \mathrm{~s}$. A meteor passed from half way between Alpha and Beta of Capricornus to Delta of Sagittarius.

"2. $9 h .30 \mathrm{~m}$. From Beta of Sagittarius to Alpha of Delphinus. The course of this was nearly upward.

"3. 10h. 18m. 34s. From one degree below Beta Aquarius to Epsilon of Sagittarius, nearly parallel to the first.

"4. 10h. 40m. From Eta of Draco to Epsilon of Corona. This was a brilliant meteor, leaving a phosphorescent train after it for a few seconds. These observations were noted by Carey's nine-inch globe of 1816. I was surprised to discover that most of these meteors had such apparent motions as would be pro. duced by bodies moving parallel to each other in straight lines. That is, they describe parts of great circles, which, if produced, would all meet and cut each other in two opposite points, like the meridians of a globe cutting each other at the poles. They appeared to move from a point in the north-east above the horizon to an opposite one in the south-west, below the horizon. By tracing the track of the above observations on the globe, the radiating point or pole was found near the star Algol, in the constellation Perseus, and the opposite, or convergent point, in the constellation Lupus. This was the course of most of the meteors. Others again, as the $2 d$, liad a course nearly at right angles to these. But I saw none which was not referrible to one of these two courses. The poles did not appear to move with the earth, but they retained their places amongst the fixed stars. Are these phenomena, as suggested by Professor Olmsted, indeed celestial in their origin, and independent of the earth's rotation?

"Yours, \&c.

"JOHN LOCKE."

"Mr. EDITOR,

"METEORS, No. II. (Ibid., Aug. 12th.)

"Since the 8th I have continued my observations on the 9th and 10th. The results are as follow :-

"On the 9 th many other meteors were seen, but not noted. No common point of radiation or convergence was ascertained.

"Aug. 10. 1st Obs. 9h. 12m. A meteor passed above, and very near to Beta of Libra, and thence ob. liquely downward, below and near to Gamma of the same.

" $2 \mathrm{~d}$ Obs. $9 h .14 \mathrm{~m} .20 \mathrm{~s}$. From $m$ of Antinous to $\mathrm{Mu}$ of Sagittarius, downward in the eastern edge of the milky way.

"3d Obs. $9 h .18 m .29 s$. From Beta of Aquarius to Psi of Capricornus.

" 4 th Obs. $9 h .26 m$. From Zeta of Serpentarius to Sigma of the same. Course downwards along the western edge of the milky way. The course of all these, as well as that of all others observed this evening, was towards one common point in the constellation of Ara. This point was south about eighteen degrees west, and fifteen degrees below the horizon.

"JOHN LOCKE."

ERR A T A.

Page 102, Table IV., heading of the second and third columns, for " $\odot$ " read " $\oplus . "$ Page 93, line 12th, for "August" read "Septembera" 







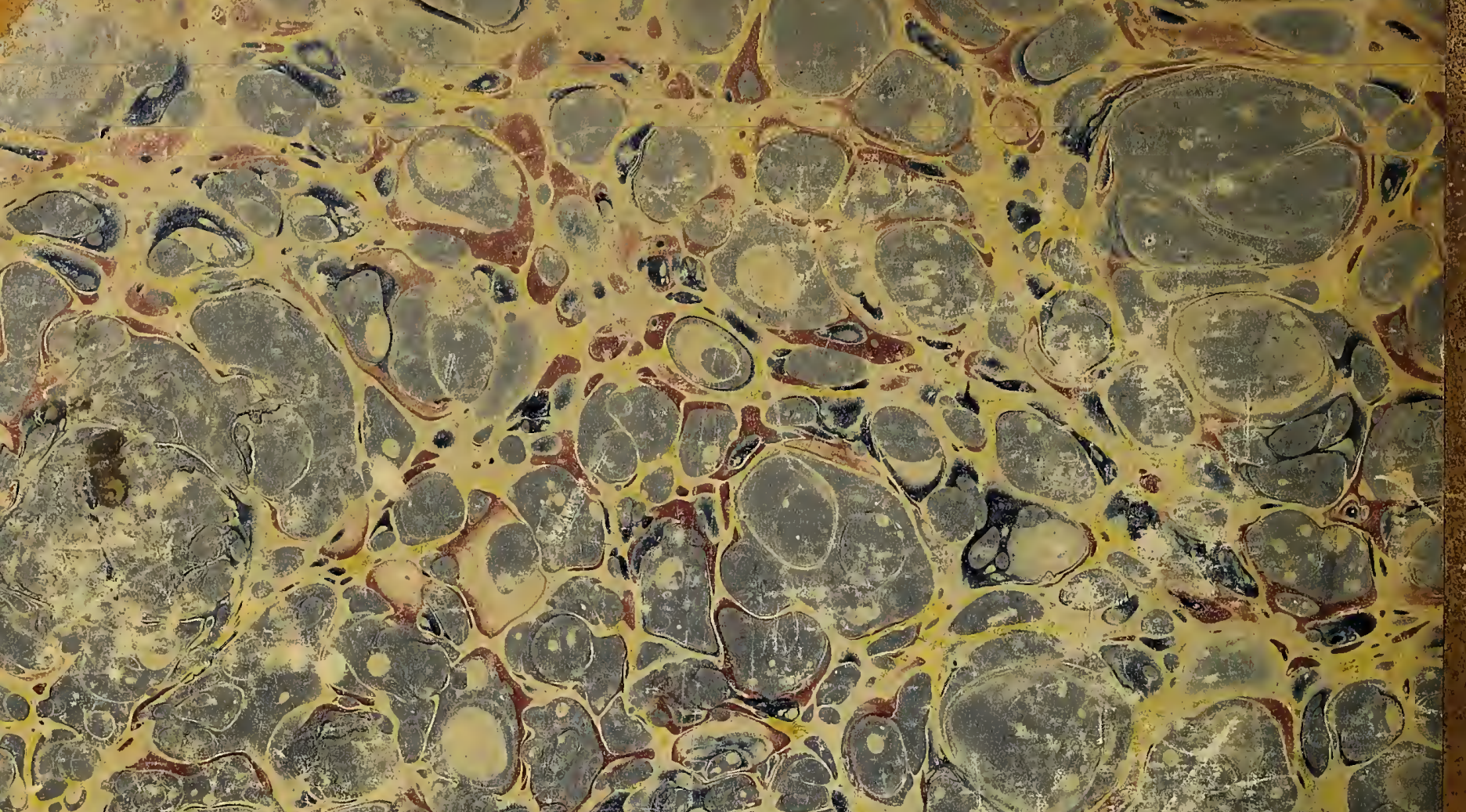

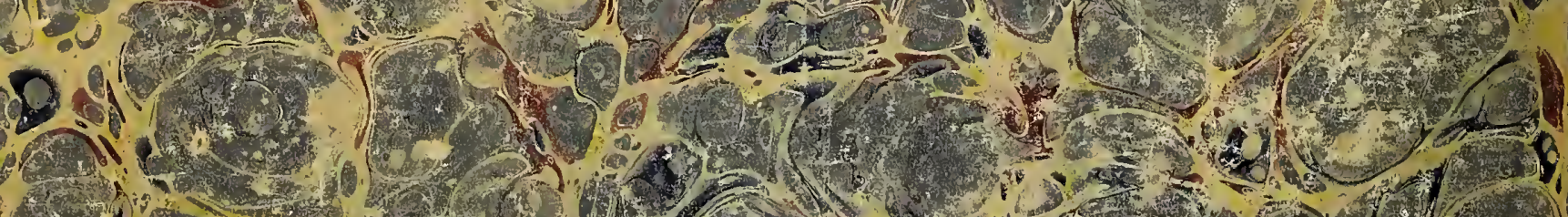
H. $602 \mathrm{mat}$ 2.0.

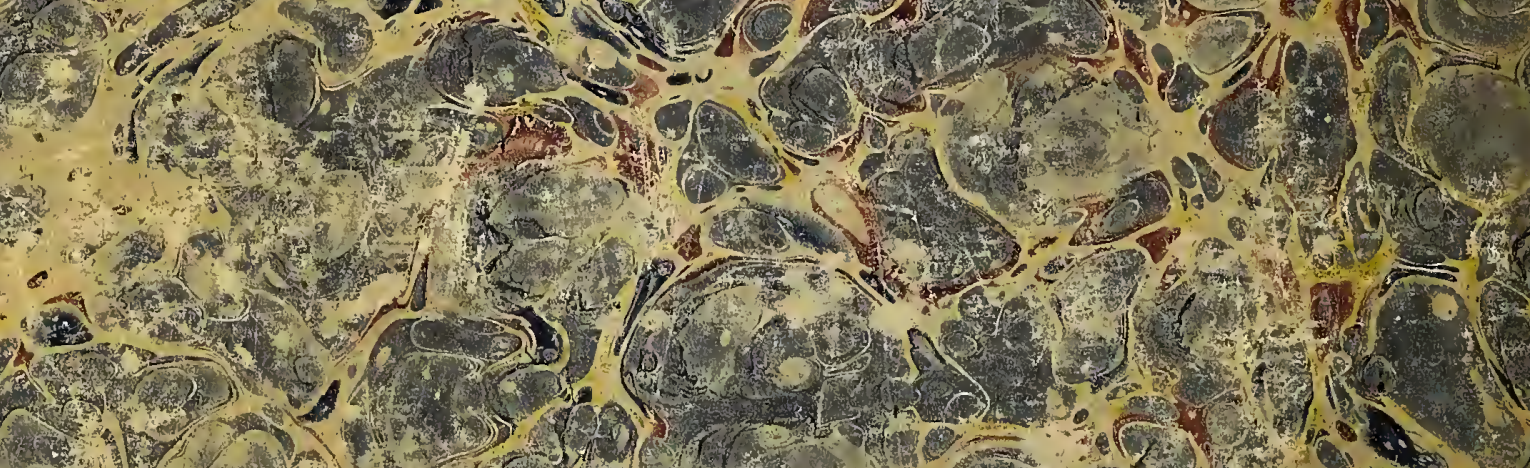

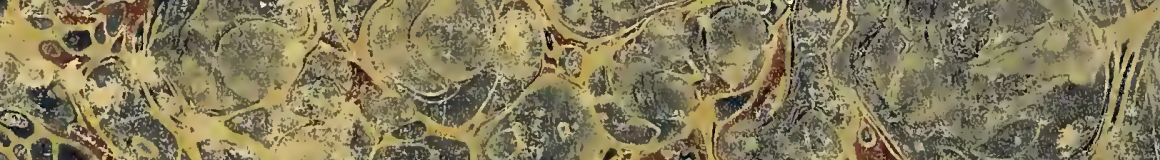

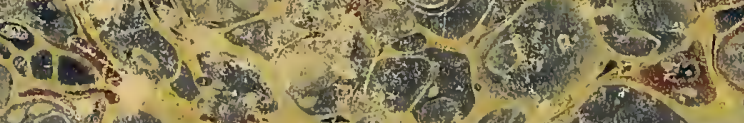

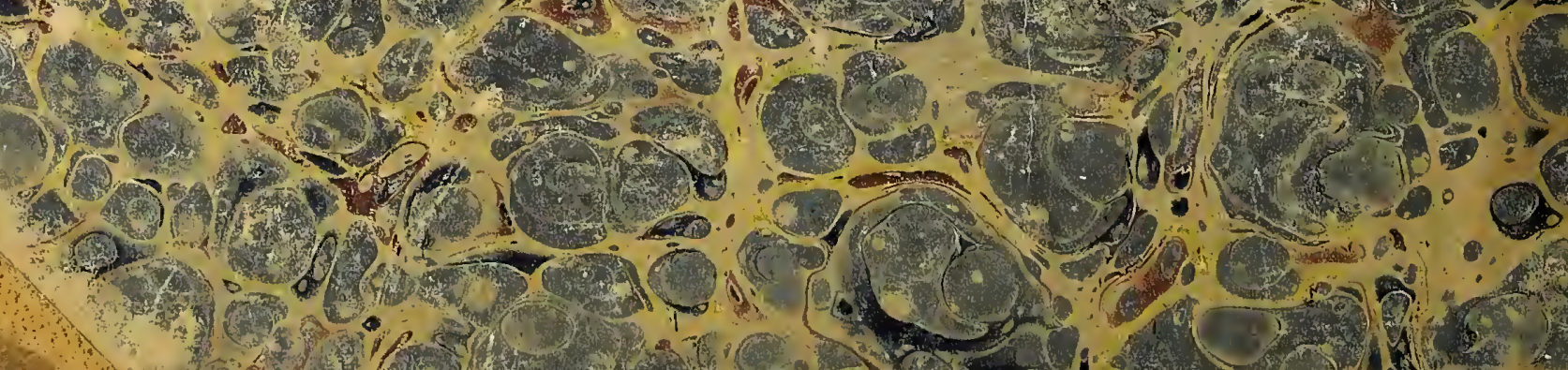
the

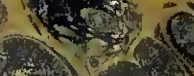

